\title{
BLCKLING BEHAVIOR OF COMPRESSION-LOADED QUASI-ISOTROPIC CURVED PANELS WITH A CIRCULAR CUTOUT
}

\author{
Mark W. Hilburger \\ National Research Council \\ Washington, D. C. 20418 \\ Vicki O. Britt \\ Gulf Stream Aerospace Corp. \\ Savannah, GA 31402-2206 \\ Michael P. Nemeth \\ NASA Langley Research Center \\ Hampton, Virginia 23681-2199
}

Presented at the 40th AIAA/ASME/ASCE/AHS/ASC Structures,

Structural Dynamics, and Material Conference

AlAA Paper No. 99-1279

St. Louis, Missouri

April 12-15, 1999 


\title{
BUCKLING BEHAVIOR OF COMPRESSION-LOADED QUASI-ISOTROPIC CURVED PANELS WITH A CIRCULAR CUTOUT
}

\author{
Mark W. Hilburger ${ }^{*}$ \\ National Research Council \\ Washington. D.C. 20418 \\ Vicki O. Britt \\ Gulf Stream Aerospace Corp. \\ Savannah, GA 31402-2206 \\ Michael P. Nemeth \\ NASA Langley Research Center \\ Hampton, Virginia 23681-0001
}

\begin{abstract}
Results from a numerical and experimental study of the response of compression-loaded quasi-isotropic curved panels with a centrally located circular cutout are presented. The numerical results were obtained by using a geometrically nonlinear finite element analysis code. The effects of cutout size, panel curvature and initial geometric imperfections on the overall response of compression-loaded panels are described. In addition, results are presented from a numerical parametric study that indicate the effects of elastic circumferential edge restraints on the prebuckling and buckling response of a selected panel and these numerical results are compared to experimentally measured results. These restraints are used to identify the effects of circumferential edge restraints that are introduced by the test fixture that was used in the present study. It is shown that circumferential edge restraints can introduce substantial nonlinear prebuckling deformations into shallow compression-loaded curved panels that can results in a significant increase in buckling load.
\end{abstract}

\section{Introduction}

A common structural element that is found in many aerospace structures is the thin-walled curved panel with a centrally located circular cutout. Cutouts commonly appear in curved panels as access ports, doors, or windows. During operation, these structural elements may experience compression loads, and thus their buckling and postbuckling behavior are important factors in determining safe operating conditions and effective designs for these structures.

Several studies have been presented that show that a circular cutout in a compression-loaded composite flat

\footnotetext{
*Post-doctoral Fellow: Member. AlaA.

† Technical Specialist. Member. AlAA. Formerly with NASA Langley Rescarch Center.

\$ Senior Research Engineer. Structural Mechanics Branch. Associale Fellow, AIAA

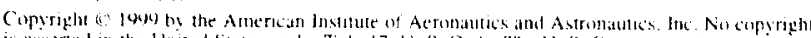

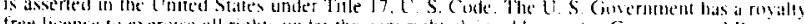

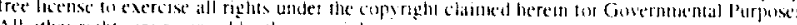
Alt other rightes are resersed b! the copyright ow fict
}

plate can have a significant effect on the buckling and postbuckling response of the structure. ${ }^{1-3}$ However, significantly fewer studies have been presented for compression-loaded curved panels with a cutout. More specifically, limited results on the effects of a circular cutout on the buckling response of compression-loaded aluminum curved panels are presented in Ref. 4 and limited results on the effects of rectangular cutouts and laminate stacking sequence on the buckling behavior of compression-loaded curved composite panels are presented in Refs. 5 through 8 . In Ref. 4 it has been shown that circular cutout size greatly affects the buckling characteristics of compression-loaded curved isotropic panels. In particular, the results indicate that aluminum panels with cutout-diameter-to-panel-width ratios $\mathrm{d} / \mathrm{W}$ ranging from 0 to 0.3 exhibit nonlinear behavior that includes a distinct buckling event with an unstable postbuckling response while panels with values of $d / W$ ranging from 0.4 to 0.6 exhibit a stable monotonically increasing nonlinear load-shortening response. These results also indicate that as the value of $d / W$ increases, the magnitude of the nonlinear prebuckling out-of-plane deformations increases, which results in an increase in the nonlinearity of the prebuckling load-shortening response curve.

A review of the results in Refs. 1 through 6 indicates that the buckling and postbuckling behavior of compression-loaded flat plates with a circular cutout is, for the most part, well understood. However, the interaction between circular cutout size, panel curvature, material orthotropy and anisotropy, initial geometric imperfections and boundary conditions, and their effects on the buckling and postbuckling response of compression-loaded composite curved panels are not well understood.

Because the amount of information on this important subject is so limited, the results from a numerical and experimental investigation of the response of compression-loaded $\mid \pm 45 / 0 / 90]_{3 .}$ quasi-isolropic curved panels with a centrally located circular cutout are presented 
herein. The objective of the present paper is to identify the effects of cutout size, panel curvature, initial geometric imperfections and selected boundary conditions on the compression response of these common laminated composite structures. Toward that objective. numerically predicted and experimentally measured results are presented and compared for 15-and 60-in-radius panels with cutout-diameter-to-plate-width ratio $d / W$ ranging from 0 to 0.6 . In addition, numerically predicted results are presented that show the effect of circumferential restraint of the loaded edges on the severity of nonlinear prebuckling deformations and the buckling load level. First, the finite-element models and analysis methods are described. Next, the test specimens and test procedure are presented. Then, an in-depth discussion of the results is given.

\section{Finite-Element Model and Analysis Methods}

A typical finite-element model of a curved panel with a centrally located circular cutout is illustrated in Fig. 1. Points on the panel mid-surface are located by an $x-y-z$ curvilinear coordinate frame whose origin is at the top corner of the panel. The panel length, width and radius are defined as $L, W$ and $R$, respectively, and the cutout diameter $\mathrm{d}$ is defined as the diameter of a right circular cylinder that intersects the panel and whose axis is perpendicular to the tangent plane at the center of the panel. Panels with 15 - and 60-in. radii were studied in the present investigation. The panel lengths and arcwidths were $14.75 \mathrm{in}$. and $14.5 \mathrm{in}$., respectively, for all panels. Cutout diameters ranged from 0 to 8.4 in. The panels were modeled as 24 -ply-thick $[ \pm 45 / 0 / 90]_{3 \mathrm{~s}}$ quasi-isotropic graphite-epoxy laminates with a nominal ply thickness of 0.005 in. and a total, nominal panel thickness of $0.12 \mathrm{in}$. The nominal lamina material properties are as follows: longitudinal modulus $E_{1}=17.5$ Msi, transverse modulus $\mathrm{E}_{2}=1.51 \mathrm{Msi}$, in-plane shear modulus $\mathrm{G}_{12}=0.78 \mathrm{Msi}$, and major Poisson's ratio $v_{12}=$ 0.295 . Idealizations of the test fixture support conditions were used in the finite-element model. To simulate clamped loaded edges, the circumferential and out-ofplane displacements, $v$ and $w$, respectively, were set equal to zero in the boundary regions of the finite-element model that extend $7 / 16$ in. in from both loaded ends of a panel, as illustrated in Fig. 1. The compression load was introduced into the panel by applying a uniform enddisplacement $\Delta$ to one end of the model while holding the other end of the panel fixed on the boundary as illustrated in Fig. 1: that is, $u\left(0, y^{\prime}\right)=\Delta$ and $u\left(L, y^{\prime}\right)=0$. The simply supported boundaries on the unloaded edges (knife-edge supports) were simulated by setting the out-of-plane displacement $w$ equal to zero on a line $5 / 16$ in. in from each unloaded edge of a panel. The finite element mesh was defined by using user-written subroutines that ate com- patible with the STAGS finite-element code. ${ }^{9}$ These user-written subroutines facilitated the generation of models with various cutout sizes and mesh densities. and provided a convenient means for assessing the convergence of a given finite-element model. Convergence were done for all finite-element models and a typical converged finite-element mesh is shown in Fig. 1.

Geometrically perfect and imperfect panels were studied in the present investigation. Nominal geometry, laminate thickness and lamina material properties were used for geometrically perfect models. Geometrically imperfect models included measured initial geometric imperfections, average measured panel thickness and thickness-adjusted material properties. The measured initial geometric imperfections were included in the model by applying a corresponding perturbation to the initial geometry of the model by using user-written subroutines. The lamina material properties were adjusted for each model using the rule of mixtures and the appropriate average measured panel thickness.

The predicted nonlinear response of the panels was determined with the STAGS finite-element code by using a combination of an arc-length path following method to model the quasi-static portions of the response and a transient analysis method to model unstable portions of the response associated with mode shape changes that occur during a buckling process. A typical finite-element model contained approximately 10,000 degrees of freedom and a typical nonlinear analysis required about 3,000 CPU seconds on an SGI Origins 2000 work station.

\section{Experiments}

A typical curved panel specimen with a centrally located circular cutout is shown in Fig. 2 and its corresponding geometry is shown in Fig. 1. The panels that were tested in the study had the same nominal dimensions and laminate properties as the models described in the previous section. The cutouts were machined in the panels using a diamond-tip machine tool. The loaded edges of the panels were machined flat and parallel in order to provide uniform end loading during the tests.

Initial geometric imperfections were measured on one side of the panel in order to characterize the actual mid-surface geometry of each specimen. The surface measurements were taken over a uniform grid that consisted of $(0.125$-in. increments along the panel $x$-axis and 0.125 -in. increments along the panel circumference. The maximum magnitude of the measured imperfections, for all panels, was on the order of $10 \%$ of the nominal panel thickness. In addition, panel thickness measurements were taken at several points on the panel to establish the average thickness of each pand.

The panels were loaded in compression by applying a uniform end-shortening to the top curved edge of 
each specimen. The loaded edges were clamped and the unloaded edges were supported by knife-edges to simulate simply supported edges. Electrical resistance strain gages were used to measure strains, and direct current differential transformers (DCDT's) were used to measure axial end-shortening and selected out-of-plane displacements of a panel. In addition. a shadow moirè technique was used to monitor out-of-plane displacements and the observed moirè fringe patterns were recorded by using video and still photography. All specimens were loaded to failure.

\section{Results and Discussion}

Numerically predicted and experimentally measured results for the compression-loaded 15- and 60-inradius panels with a circular cutout are presented in this section. The predicted results that are presented subsequently were obtained from finite-element models that include the appropriate measured initial geometric imperfections, unless it is specified otherwise. First, results are presented for the 15 -in-radius panels and then similar results are presented for 60 -in-radius panels. Finally, results that show the effects of elastic circumferential loaded-edge restraint on the prebuckling and buckling response of a geometrically perfect 60 -in-radius panel with no cutout are presented to explain some anomalies that were encountered in the test-analysis correlation. The values of axial load $\mathrm{P}$ and various displacement quantities, presented herein, are normalized with respect to the corresponding linear bifurcation buckling load of a panel without a cutout $\mathrm{P}_{\mathrm{cr}}^{\mathrm{O}}$, that was obtained from a finite-element analysis, and the nominal panel thickness $t$ $=0.12$ in., respectively, unless it is specified otherwise. In addition, values of axial stress resultant $N_{x}$ are normalized with respect to the corresponding linear bifurcation buckling stress resultant $\mathrm{N}_{\mathrm{x}}{ }^{\circ} \mathrm{cr}$ which is equal to $\mathrm{P}_{\mathrm{cr}}^{0}$ $\mathrm{W}$. The values of $\mathrm{P}_{\mathrm{cr}}^{\mathrm{r}}$ that were obtained for the 15- and 60 -in-radius panels are 66.49 kips and 17.44 kips, respectively.

\section{5-in-Radius Panels}

Typical predicted and measured load-shortening response curves for the 15 -in-radius panels with various size cutouts are shown in Fig. 3. Two groups of seven curves are shown in Fig. 3 that correspond to values of $0 \leq \mathrm{d} / \mathrm{W} \leq 0.6$. The solid curves correspond to the measured experimental results and the dashed curves correspond to the predicted results. Buckling points are marked by filled squares and circles for the measured and predicted response curves, respectively. An " $x$ " indicates the failure of a test specimen.

The measured load-shortening response curves for the 15-in-radius panels show good correlation with the corresponding predicted responses, for the full range of cutout sizes. More specifically. the maximum difference between predicted and measured values of initial prebuckling stiffness is $3 \%$ and the maximum difference between predicted and measured buckling load values is $5 \%$, which occurs for the panel with $\mathrm{d} / \mathrm{W}=0.1$. For the panels with $d / W=0.1$ and 0.2 , however, the unstable buckling events resulted in catastrophic failure of the panels and thus resulted in no residual postbuckling strength. These two catastrophic failures appear to be caused by material compression-type failures that initiated near high strain concentrations on the free edge of the cutout and then propagated almost instantaneously to the outer edge of the panel during the failure event. The panels with values of $\mathrm{d} / \mathrm{W}=0,0.3,0.4$, and 0.5 have various levels of residual postbuckling strength and can thus sustain additional load before failure occurs. For the panel with $\mathrm{d} / \mathrm{W}=0$, the panel failed near the intersection between the loaded-boundary fixture and the knife-edge support because of a high strain concentration in that region. For values of $\mathrm{d} / \mathrm{W}=0.3,0.4,0.5$, and 0.6 , the panel failures appear to be caused by an interlaminar shear failure near the cutout that is caused by large out-of-plane bending deformations that occurred near the cutout.

The results in Fig. 3 indicate that a cutout has a significant effect on the prebuckling, buckling and postbuckling responses of the compression-loaded quasiisotropic curved panel considered herein. In particular, the predicted and measured results indicate that a significant increase in cutout size results in a significant decrease in the overall effective prebuckling stiffness of the panel and a substantial increase in the degree of nonlinearity of the prebuckling load-shortening response curve, just like the aluminum panels of Ref. 4 . The measured and predicted results also indicate that for values of $\mathrm{d} / \mathrm{W}$ ranging from 0 to 0.3 , the panels have well defined buckling points (marked by a filled circle and square symbols in the figure) and show an unstable buckling event that exhibits a significant reduction in axial load and a distinct change in the panel displacement field. However, for the panels with values of $\mathrm{d} / \mathrm{W}=0.1$ and 0.2 , the buckling points are marked with an " $x$ " to indicate that the buckling event also coincided with the failure of the panel. In addition, as the value of $\mathrm{d} / \mathrm{W}$ increases from 0.1 to 0.3 , the buckling load decreases monotonically. However, the buckling load for a panel with $\mathrm{d} / \mathrm{W}=0$ is about $5 \%$ less than that for the panel with $\mathrm{d} / \mathrm{W}=0.1$. For values of $\mathrm{d} / \mathrm{W}=0.4$ through 0.6 , the results show that the response no longer exhibits an unstable buckling event. For the panels with $\mathrm{d} / \mathrm{W}=0.4$ and 0.5 , the measured results indicate that each of the panels reach a corresponding limit point that is followed by relatively benign decrease in load before additional load can be applied to the panels. This benign limit-point behavior is accompanied by a gradual change in panel displacements instead of an intense, dymamic change in the displacement field like that exhibited by panels with small values of $\mathrm{d} / \mathrm{W}$. For the 
panel with $\mathrm{d} / \mathrm{W}=0.6$, the response is characterized by a monotonically increasing load-shortening response curve that does not exhibit a buckling event. For panels with these cutout sizes, large out-of-plane deformations develop near the cutout at the onset of loading and their amplitudes grow monotonically in a stable manner throughout the loading. The benign behavior that is exhibited by the panels with $0.4 \leq \mathrm{d} / \mathrm{W} \leq 0.6$ is unlike the behavior shown for flat plates with the same values of $d /$ $\mathrm{W}$ (see Ref. 8). In addition, the corresponding linear bifurcation buckling loads no longer have any phy'sical significance since the panels no longer exhibit a buckling response nor do they exhibit a sudden change in effective axial stiffness associated with a buckling event.

Numerically predicted and experimentally measured effective initial prebuckling stiffnesses and buckling loads for the 15-in-radius panels are summarized in Figs. 4 and 5, respectively. The effective initial prebuckling stiffnesses are defined as the initial slope of the loadshortening curves in Fig. 3. Three groups of results are presented in Figs. 4 and 5. The solid curves correspond to predicted results from geometrically imperfect models, the dashed lines correspond to results from geometrically perfect models, and the filled squares correspond to experimentally measured results.

The results in Figs. 4 and 5 show good agreement between analysis and experiment and indicate a reduction in the prebuckling stiffness of about $54 \%$ as the value of $\mathrm{d} / \mathrm{W}$ increases from 0 to 0.6 . Similarly, the results indicate a $53 \%$ reduction in the buckling load as $\mathrm{d} / \mathrm{W}$ increases from 0.1 to 0.5 . Moreover, the results indicate, for the most part, that the inclusion of the measured imperfections in the numerical models have essentially no effect on the predicted initial prebuckling stiffness for $0 \leq \mathrm{d} / \mathrm{W} \leq 0.6$ and the value of the predicted buckling load for $0.1 \leq \mathrm{d} / \mathrm{W} \leq 0.4$. Predicted results for the panel with $\mathrm{d} / \mathrm{W}=0$, however, indicate about a $14 \%$ reduction in the predicted buckling load because of the initial geometric imperfections. Moreover, the predicted results for $\mathrm{d} / \mathrm{W}=0$ and 0.1 , that include measured initial geometric imperfections, show the same trend as the measured results; that is, the panel with $d / W=0.1$ has a higher buckling load than the panel with $\mathrm{d} / \mathrm{W}=0$. The predicted and measured results show about a $4.8 \%$ and $5.3 \%$ increase, respectively, in the buckling load as $\mathrm{d} / \mathrm{W}$ increases from 0 to 0.1 . These results at first glance suggest that the buckling load of the panel with $d / W=0$ may be more sensitive to initial geometric imperfections than the panel with a value of $d / w=0.1$. However, examination of the measured initial geometric imperfections for these two panels showed that the panel with $\mathrm{d} / \mathrm{W}=0$ had a maximum imperfection amplitude that was nearly twice that of the panel with $\mathrm{d} / \mathrm{W}=0.1$.

Measured and predicted prebuckling out-of-plane displacement $\delta$ at a point on the edge of the cutout are presented in Fig. 6 where $\delta \approx w(L / 2 . W / 2+d / 2)$. Two groups of seven curves are shown in Fig. 6 that correspond to $0 \leq \mathrm{d} / \mathrm{W} \leq 0.6$. The solid curves correspond to the measured experimental results and the dashed curves correspond to the predicted results. These results indicate that, as the size of the cutout increases, the nonlinear prebuckling displacements increase dramatically. This trend is consistent with the increase in the nonlinearity of the prebuckling load-shortening response curves with increasing cutout size that is shown in Fig. 3. The predicted results for panels with larger values of $d /$ W agree well with measured results, however, correlation between predicted and measured results for panels with small values of $d / W$ is poor.

Predicted out-of-plane displacement contours and observed moirè fringe patterns for 15 -in-radius panels with $\mathrm{d} / \mathrm{W}=0,0.2$, and 0.4 are shown in Figs. 7, 8, and 9, respectively. Because of the curvature of the panels, the observed moirè fringe patterns that are presented are somewhat distorted, however, some common features of the predicted displacement contours and the observed fringe patterns can be identified. The dashed contour lines in the predicted contours represent inward displacements and the solid lines represent outward displacements. The density of the contour lines is indicative of the severity of the displacement gradients in the panel.

Moirè fringe patterns for a panel with $d / W=0$ at the onset of buckling and initial postbuckling points are shown in Figs. $7 \mathrm{a}$ and $7 \mathrm{~b}$, respectively. The fringe pattern at the onset of buckling indicates slight inward displacements in the upper right corner of the panel. Upon buckling, the panel snaps dynamically into a mode shape that consists of one half-wave along the panel generator and two half-waves across its circumference. The predicted postbuckling displacement contours, shown in Fig. $7 \mathrm{~d}$, correlate well qualitatively with the observed fringe patterns. It is difficult, however, to determine if the predicted displacement contours at the onset of buckling, shown in Fig $7 c$, correlate well with the observed fringe pattern because of the distortion effects of the panel curvature on the pattern and the lower density of the fringes.

Observed fringe patterns for a panel with $\mathrm{d} / \mathrm{W}=0.2$ at the onset of buckling and initial postbuckling points are shown in Figs. $8 \mathrm{a}$ and $8 \mathrm{~b}$, respectively. Predicted results for the buckling and initial postbuckling displacement contours are show'n in Figs. $8 \mathrm{c}$ and $8 \mathrm{~d}$, respectively. The predicted buckling displacement contours agree well with the observed fringe pattern in Fig. 8a. These results indicate that, at buckling, the panel exhibits relatively large out-of-plane hending displacements near the edge of the cutout. Buckling of this panel resulted in caltastrophic failure of the panel (hence $P / P^{0}{ }^{\circ}=(0)$, thus the fringe pattern is not associatted with a postbuckling mode shape. It is. however, qualitatively similar to the intial 
postbuckling displacement contour shoun in Fig. 8d.

The observed buckling and initial postbuckling displacement fringe patterns for a panel with $\mathrm{d} / \mathrm{W}=0.4$ are shoun in Figs. 9a and 9b, respectively. The observed displacements at buckling consist of large outward bending deformations near the cutout edge and several inward buckles away from the cutout. As loading continues in the postbuckling range, the displacement fringe pattern in Fig. 9a exhibits a gradual change into the fringe pattern shown in Fig. 9b that consists of one large inward buckle to the left of the cutout while retaining a fringe pattern on the right side of the panel that is similar to the corresponding region in Fig 9a. This observed change in the displacement field happens in a stable, benign manner. The predicted results in Figs. $9 c$ and $9 d$ indicate good qualitative agreement between the observed fringe patterns and the predicted displacement contours.

The predicted fringe patterns that are shown in Figs. 7-9 exhibit essentially either reflective symmetry or antisymmetry about the $\operatorname{arc} x=L / 2$ or the line $y=W /$ 2. Similar contours for the corresponding geometrically perfect panels indicate that the small amount of deviation from these symmetries are caused by the small, asymmetric initial geometric imperfections and not by flexural anisotropy. This fact is consistent with the small values of the anisotropy parameters ( $g=d=0.04$ ), for the laminate considered herein, that are presented in Ref. 10.

Predicted stress resultant distributions on the end $(x$ $=0$ ) of a 15-in-radius geometrically imperfect panel with three different cutout sizes and for various load levels are shown in Figs. 10,11 and 12. The stress resultant $N_{x}$ is normalized with respect to the linear-bifurcation buckling stress resultant of a corresponding panel without a cutout $\mathrm{N}_{\mathrm{x}}{ }^{\mathrm{O}} \mathrm{cr}$, and the circumferential coordinate $\mathrm{y}$ is normalized with respect to the panel arc-width $W$ such that $0 \leq y / W \leq 1.0$. Three curves are shown in each of the figures that correspond to predicted stress resultant distributions for panels three different values of $d / W$. The solid curves correspond to results for $\mathrm{d} / \mathrm{W}=0$, the dashed curves correspond to results for $\mathrm{d} / \mathrm{W}=0.2$ and the dashdotted curves correspond to results for $\mathrm{d} / \mathrm{W}=0.4$.

Predicted prebuckling stress resultant distributions for panels with $\mathrm{d} / \mathrm{W}=0,0.2$, and 0.4 at a value of $\mathrm{P} / \mathrm{P}^{\circ}$ cr $=0.5$ are presented in Fig. 10. These results indicate that, at this load level. the stress distribution for a panel with $\mathrm{d} / \mathrm{W}=0$ is, mostly, uniform and symmetric about the line $y / W=0.5$. The sawtooth shape at the far edges of the distribution is caused by the simulated knife-edge supports and is an artifice of the localized bending of a panel near the knife-edge supports. As the value of $\mathrm{d} / \mathrm{W}$ increases the load is redistributed towards the edge of the panel in a symmetric manner. The predicted stress resultant distributions at the onset of buckling for panels with $\mathrm{d} / \mathrm{W}=$ 0. 0.2. and (1.4 are shown in Fig. 11. In this figure, the onset of buckling corresponds to $\mathrm{P} / \mathrm{P}_{\mathrm{cr}}^{0}=0.77 .0 .57$, and 0.40 for panels with $\mathrm{d} / \mathrm{W}=0,0.2$. and 0.4 , respectively. These results and the results in Fig. 10 indicate that the stress resultant distribution for the panels with $d / W=0$ and 0.2 remains symmetric up to buckling with only an overall increase in the magnitude of the stress resultant. However, for the panel with $\mathrm{d} / \mathrm{W}=0.4$, the stress resultant distribution changes considerably when the buckling load is approached, which is seen by comparing the corresponding results in Figs. 10 and 11. More specifically, the central region of the panel edge becomes practically unloaded and the stress resultant distribution become asymmetric as the buckling load is approached. Unloading of the central region of the panel is caused by increasingly large prebuckling out-of-plane bending deformations near the cutout (as shown in Fig. 9c) that diminish the axial stiffness of the central region of the panel which cause the significant redistribution of the load towards the simply supported edges of the panel. This load path shift is consistent with the reduction in the effective stiffness of the panels that is shown in Fig. 3 as loading approaches the buckling load. The loss of symmetry of the stress resultant distribution is a result of an asymmetric growth in the nonlinear prebuckling deformations in the panel that is caused by asymmetry in the initial geometric imperfection shape.

Predicted initial postbuckling stress resultant distributions for panels with $\mathrm{d} / \mathrm{W}=0,0.2$, and 0.4 , that correspond to values of $\mathrm{P} / \mathrm{P}^{\mathrm{o}}{ }_{\mathrm{cr}}=0.53,0.43$ and 0.39 , respectively, are presented in Fig. 12. The stress distributions for the panels with $\mathrm{d} / \mathrm{W}=0$ and 0.2 are significantly different from the corresponding stress distribution at the onset of buckling (see Fig. 11) because of the substantial asymmetry in the initial postbuckling displacement fjelds (see Figs. 7-9) and the redistribution in load that accompanies buckling. For these panels, the stress distributions exhibit minimum and maximum stress values associated with large inward and outward buckles in the panel, respectively, as shown in Fig. 7d and $8 \mathrm{~d}$. The initial postbuckling stress resultant distribution for the panel with $\mathrm{d} / \mathrm{W}=0.4$ is similar to the stress resultant distribution at the onset of buckling (see Fig. 11), however, the minimum stress resultant value decreases somewhat upon the formation of a large buckle on one side of the cutout, as seen in Fig. 9d. In addition, it is interesting to note that the minimum stress values for the panels with $\mathrm{d} / \mathrm{W}=0.2$ and 0.4 have reversed sign

\section{0-in-Radius Panels}

Numerically predicted and experimentally measured load-shortening response curves for the $6($ )-in-radius panels are presented in Fig. 13. Two groups of seven curves are shown in Fig. 13 that correspond to values of $0 \leq \mathrm{d} / \mathrm{W} \leq 0.6$. The solid curves correspond to measured experimental results and the dashed curves corre- 
spond to the predicted results. Buckling points are marked by filled squares and circles for the measured and predicted response curves, respectively. An " $x$ " indicates the failure of a test specimen.

The results in Fig. 13 indicate that the measured and predicted trends associated with the effects of cutout size on the load-shortening response of a 60 -in-radius panel correlate well for the larger values of $d / W$. However, there is a large discrepancy in the results for the smaller values of $d / W$, especially for $d / W=0$. In particular, the measured buckling load for $\mathrm{d} / \mathrm{W}=0$ is almost $30 \%$ greater than the predicted buckling load. In contrast, the measured buckling loads for $\mathrm{d} / \mathrm{W}=0.1$ and 0.2 are greater than the predicted buckling loads by $7 \%$ and $2 \%$, respectively.

The measured and predicted results in Fig. 13 indicate the panels with values of $\mathrm{d} / \mathrm{W}=0$ to 0.2 exhibit a significant amount of prebuckling nonlinearity and have well defined buckling points that are marked by the filled symbols in the figure. Moreover, the prebuckling nonlinearity in the load-shortening response curves is much more pronounced for the 60 -in-radius panels than for the 15-in-radius panels. An unstable postbuckling response occurs beyond the buckling point for each panel that is characterized by a reduction in axial load and a distinct dynamic change in the panel displacement field. In addition, as the value of $\mathrm{d} / \mathrm{W}$ increases, the buckling load decreases monotonically. Moreover, the panels sustain additional load that is substantially larger than the initial postbuckling load until catastrophic failure occurs, which is marked by an " $x$ " in the figure. Unlike flat plates, the results indicate that the panels with $d$ / $W=0.3$ to 0.6 no longer exhibit a buckling event; rather, the response is characterized by a monotonically increasing load-shortening response curve up to failure. The predicted and measured results indicate that the panels exhibit a transition from a buckling response to a monotonically increasing stable response between $\mathrm{d} / \mathrm{W}=0.2$ and 0.3 , unlike the results for the 15 -in-radius panels which exhibit the transition between $\mathrm{d} / \mathrm{W}=0.4$ and 0.6 . These results indicate that curvature has a significant effect on the character of the nonlinear compression response of panels with a central circular cutout.

Another important point to note is that the predicted and measured buckling loads all have values that are much greater than the predicted linear bifurcation buckling load for the 60-in-radius panel without a cutout. For example, the panel with $\mathrm{d} / \mathrm{W}=0$ has a buckling load $75 \%$ greater than the corresponding linear bifurcation buckling load. The high buckling load values are caused by large prebuckling deformations that develop in the panel and act to retard the onset of buckling. These nonlinear prebuckling deformations are neglected in a linear bifurcation buckling analysis. Moreover, the substantial nonlinear prebuckling response that is exhibited by the panel with $\mathrm{d} / \mathrm{H}^{\prime}=0$ is contrary to known behavioral character- istics of compression-loaded shallow curved panels. These large differences in the buckling load and corresponding linear bifurcation buckling load indicate that a linear bifurcation analy'sis of a curved panel may not represent accurately the actual buckling behavior of shallow curved panels in some cases.

Two types of failure were observed for the 60-inradius panels. Panels with $\mathrm{d} / \mathrm{W}=0$ to 0.3 exhibited catastrophic compression failures near the test fixture on the loaded ends and displayed little evidence of material failures near the cutout. Panels with $\mathrm{d} / \mathrm{W}=0.4$ to 0.6 exhibited interlaminar shear failures near the free-edge of the cutout that was caused by extensive out-of-plane bending deformations in the region.

Predicted and measured effective initial prebuckling stiffnesses and buckling load values for the 60 -in-radius panels are presented in Figs. 14 and 15, respectively. The effective initial prebuckling stiffnesses in this figure are defined as the initial slope of the load-shortening curves in Fig. 13. Three groups of results are presented in Figs. 14 and 15. The solid curves correspond to predicted results from geometrically imperfect models, the dashed lines correspond to results from geometrically perfect models, and the filled squares correspond to the experimentally measured results.

The results in Fig. 14 show good correlation between the predicted and measured trends and indicate a reduction in initial prebuckling stiffness of about $48 \%$ as $\mathrm{d} / \mathrm{W}$ increased to 0.6 . The measured imperfections are shown to have no influence on the effective prebuckling stiffness of the panels. The results in Fig. 15 show good correlation between predicted and measured values of the buckling loads for $\mathrm{d} / \mathrm{W}=0.1$ and 0.2 , and poorer correlation for $d / W=0$. Overall, all the results indicate a reduction in buckling load as $\mathrm{d} / \mathrm{W}$ increases from 0 to 0.2 . Unlike the results for the effective initial prebuckling stiffness, the addition of measured imperfections for $\mathrm{d} / \mathrm{W}$ $=0$ results in a $5.6 \%$ reduction in the buckling load with respect to the corresponding buckling load for the geometrically perfect panel, while the imperfections for $\mathrm{d} / \mathrm{W}$ $=0.1$ and 0.2 result in a $1 \%$ and $0.5 \%$ increase in the buckling load, respectively. This trend, and the fact that the amplitudes of the measured initial geometric imperfections for these panels are nearly the same, indicates that the buckling load of a panel with a small value of $d /$ $\mathrm{W}$ is more sensitive to initial geometric imperfections than the panels with the larger values of $d / W$. Moreover, the panels with $\mathrm{d} / \mathrm{W}>0.2 \mathrm{do}$ not exhibit a buckling event and, as a result, do not exhibit imperfection sensitivity.

A comparison between numerically predicted and experimentally measured out-of-plane displacements $\delta$ at a point on the edge of the cutout is presented in Fig. 16 where $\delta \approx w(1 / 2 . w / 2+\mathrm{d} / 2)$. Two groups of seven curves are shown in Fig. 16 that correspond $100 \leq \mathrm{d} / \mathrm{W}$ $\leq 0.6$. The solid curves correspond to the measured ex- 
perimental results and the dashed curves correspond to the predicted results.

Like the load-shortening results in Fig. 13, the results in Fig. 16 indicate that, as the cutout size increases, the nonlinear prebuckling displacements increase dramatically. In addition. it is interesting to note that the magnitudes of the predicted and measured prebuckling out-of-plane displacements are significantly larger for the 60 -in-radius panels than for the 15-in-radius panels (compare Figs. 3 and 13). This trend is consistent with the greater degree of nonlinearity of the prebuckling load-shortening response curves that is exhibited by the 60 -in-radius panels, compared to the 15 -in-radius panels. For values of $\mathrm{d} / \mathrm{W}=0.5$ and 0.6 , the predicted and measured results correlate well, but as the value of $d / W$ decreases, the correlation becomes increasingly worse.

Predicted and observed out-of-plane displacement contours for $\mathrm{d} / \mathrm{W}=0,0.2$ and 0.4 are presented in Fig. 17,18 , and 19 , respectively. The dashed contour lines in the predicted contours represent inward displacements and the solid lines represent outward displacements. The density of the contour lines is indicative of the severity of the displacement gradients in the panel.

The observed moirè fringe pattern for $d / W=0$ at the onset of buckling is shown in Fig. 17a. The fringe pattern is characterized by an hour-glass-shaped outward radial displacement pattern. Upon buckling, the panel snaps through into a postbuckling mode shape that consists of a single, large centrally located inward buckle, as shown in Fig 17b. The predicted postbuckling displacement contours in Fig 17d agree well with the observed fringe pattern in Fig. 17b, however, the predicted displacement contours, just prior to buckling, in Fig 17c do not agree well with the observed fringe pattern in Fig 17a. The observed moire fringe pattern of a panel with $\mathrm{d} / \mathrm{W}=$ 0.2 at the onset of buckling is shown in Fig. 18a. The fringe pattern indicates outward out-of-plane bending of the cutout edges and inward radial displacements between the cutout and the loaded edges of the panel. The initial postbuckling-displacement fringe pattern is shown in Fig. 18b and consists of an inward buckle pattern that is similar to the pattern that is shown in Fig. $17 \mathrm{~b}$ for $\mathrm{d} / \mathrm{W}$ $=0$. The predicted displacement contours that correspond to the onset of buckling and initial postbuckling are shown in Figs. 18c and 18d, respectively, and agree well with the observed fringe patterns in Figs. 18a and 18b. The observed moire fringe pattern for $\mathrm{P} / \mathrm{P}_{\mathrm{cr}}{ }_{\mathrm{c}}=0.63$ and $\mathrm{d} / \mathrm{W}=0.4$ is presented in Fig. $19 \mathrm{a}$ and is characterized by large outward out-of-plane bending displacements at the cutout edge. The displacement field shown in Fig. 19b indicates that the bending deformations near the cutout have become increasingly severe as the loading increases. The predicted results in Fig $19 \mathrm{c}$ and $19 \mathrm{~d}$ correlate well with the observed fringe patterns in Fig $19 \mathrm{a}$ and $19 \mathrm{~b}$, respectively.

A comparison of predicted stress resultant distribu- tions on the loaded end $x=0$ of the 60 -in-radius panels with $\mathrm{d} / \mathrm{W}=0,0.2$, and 0.4 at various load levels are presented in Figs. 20,21, and 22. The stress resultant $N_{x}$ is normalized with respect to the linear bifurcation stress resultant of a panel without a cutout $\mathrm{N}_{\mathrm{x}}{ }_{\mathrm{cr}}$, and the circumferential coordinate $y$ is normalized with respect to the panel arc-width $W$ such that $0 \leq \mathrm{y} / \mathrm{W} \leq 1.0$. Three curves are shown in each of the figures that correspond to predicted stress resultant distributions for panels with $\mathrm{d} / \mathrm{W}=0,0.2$ and 0.4 . The solid curves correspond to results for $d / W=0$, the dashed curves correspond to results for $\mathrm{d} / \mathrm{W}=0.2$ and the dash-dotted curves correspond to results for $d / W=0.4$.

The predicted prebuckling stress resultant distributions for a load level of $\mathrm{P} / \mathrm{P}_{\mathrm{cr}}^{\circ}=0.5$ are shown in Fig. 20. These results indicate that, for $d / W=0$, the center of the panel and the supported edges have a slightly greater stress resultant magnitudes than the rest of the panel. As the value of $d / W$ increases, the center of the panel becomes unloaded and the load path is shifted towards the simply supported edges.

Predicted stress resultant distributions for panels $\mathrm{d} /$ $\mathrm{W}=0$ and 0.2 at the onset of buckling, that correspond to values of $\mathrm{P} / \mathrm{P}^{\circ} \mathrm{cr}=1.35$, and 1.23 , respectively, and for $\mathrm{d} / \mathrm{W}=0.4$ at $\mathrm{P} / \mathrm{P}^{\mathrm{o}}{ }_{\mathrm{cr}}=0.63$ (the load level at which the first distinct displacement pattern appears) are presented in Fig 21. The results in Figs. 20 and 21 indicate that, as the loading is increased in the prebuckling range, all the stress resultant distributions remain symmetric about the line $y / W=0.5$. Moreover, the stress resultant distributions for panels with $\mathrm{d} / \mathrm{W}=0.2$ and 0.4 remain similar in shape while increasing in overall magnitude. For $\mathrm{d} / \mathrm{W}=$ 0 , however, an increasingly larger portion of the load is redistributed towards the center of the panel. This response is caused by an increase in curvature of the panel that occurs during loading, as indicated in Fig. 17c, which causes a geometric stiffening of the panel and a shift of the load path into the stiffer central portion of the panel. As the cutout size increases, stiffness is lost in the central region of the panel which impedes the load path from being centrally located.

Predicted initial postbuckling stress resultant distributions for $\mathrm{d} / \mathrm{W}=0$ and 0.2 , that correspond to values of $\mathrm{P} / \mathrm{P}^{\circ}{ }_{\mathrm{cr}}=0.73$, and 0.74 , respectively, and for $\mathrm{d} / \mathrm{W}=0.4$ at $\mathrm{P} / \mathrm{P}_{\mathrm{cr}}^{\mathrm{O}}=0.63$ (the load level at which the second distinct displacement pattern appears) are presented in Fig 22. In all cases, extensive deformations in the central portion of each panel have diminished significantly the overall load-carrying capacity and have caused most of the load to be redistributed loward the simply supported edges of the panels. In particular, the center of the panels with $\mathrm{d} / \mathrm{W}=()$ and 0.2 , have unloaded completely.

The predicted prebuckling, buckling and postbuck- 
ling stress resultant distributions for the 60 -in-radius, geometrically imperfect panels shown in Figs. 20-22 are symmetric throughout the load history of the panels. In contrast. the predicted stress resultant distributions for the 15-in-radius panels (Figs. 10-12) are symmetric at a load level of $\mathrm{P} / \mathrm{P}^{\circ}{ }_{\mathrm{cr}}=0.5$ but become asymmetric after buckling. These differences in the stress resultant distributions of the 15- and 60-in-radius panels are a consequence of the panel curvature. More specifically, the 60 in-radius panels exhibit symmetric initial postbuckling deformations while, in contrast, the 15-in-radius panels exhibit asymmetric initial postbuckling deformations.

\section{Anomalous Behavior}

For the most part, correlation between the numerically predicted results and the experimentally measured results presented herein is good. However, significant discrepancies and unexpected behavioral characteristics were identified for some of the panels and warranted further study. Most notably, the 60 -in-radius panels exhibit a significant amount of nonlinearity in the prebuckling portion of the load-shortening response curve that is shown in Fig. 13. In addition, the 60-in-radius panels exhibit buckling load values that are significantly greater in magnitude than the corresponding predicted linear bifurcation buckling load for a panel without a cutout. These response characteristics are contrary to the known behavior of compression-loaded curved panels such as that given in Refs. 11 through 13. In all cases, the measured buckling loads for 15- and 60-in-radius panels are larger in magnitude than the corresponding predicted buckling load values. The largest discrepancy between measured and predicted buckling load values occurs for the 60 -inradius panel with $\mathrm{d} / \mathrm{W}=0$ in which case the predicted buckling load value is approximately $30 \%$ less than the measured value. Furthermore, the predicted and observed buckling deformations for the 15 - and 60 -in-radius panels with $d / W=0$ do not agree well. The observed discrepancies are generally more pronounced for panels with relatively small values of $\mathrm{d} / \mathrm{W}$, and they tend to diminish as the value of $d / W$ increases.

As a first step towards identifying the source of the significant prebuckling nonlinearity that is exhibited by the 60 -in-radius panels, the effects of the circumferential boundary conditions $v(0, y)=v(L, y)=0$ and $N_{x y}(0, y)$ $=N_{x y}(\mathrm{~L}, y)=0$ on the response were examined. The numerical results for the latter traction-free boundary condition indicated that allowing the loaded edges to expand circumferentially in an unrestricted manner eliminated the prebuckling nonlinearity of the response. Because of this result, the result that the panel with $v(0, y)=v(L, y)$ $=0$ underpredicts the degree of prebuckling nonlinearity seen in the test results, and because the test fixture actually applies some restraint to the circumferential displacements of the loaded edges of a pamel. anonher edge condition was considered. This edge condition is the case in which the circumferential displacement of the loaded edges are elastically restrained. This type of boundary condition was used as a "first-order" engineering approximation to the actual edge restraint that is caused by the test fixture. Elastic circumferential edge restraints on the loaded ends of a panel are simulated in the finite-element model by applying an externally-generated linear circumferential stiffness contribution to the nodes in the boundary regions that extend $7 / 16$ in. in from both loaded ends of the panel, as illustrated in Fig. 1. The magnitude of the stiffness component was varied from $0 \mathrm{lb} / \mathrm{in}$. to $1.0 \times 10^{8} \mathrm{lb} / \mathrm{in}$. in order to simulate circumferential restraints that range from free expansion to fixed conditions. As a point of reference, the circumferential membrane stiffness of the panels is given by $\mathrm{A}_{22}=$ $9.22 \times 10^{5} \mathrm{Ib} / \mathrm{in}$. Results that show some of the effects of elastic circumferential edge restraints on the compression response of the 60-in-radius panel with $d / W=0$ are presented in the following section.

\section{Effects of Elastic Circumferential Edge Restraints}

Load-shortening response curves for a 60 -in-radius geometrically perfect panel with various values of elastic circumferential stiffness $K_{V}$ are presented in Fig. 23 and illustrate the overall effects of an elastic circumferential edge restraint on the compression response. Five curves are shown in Fig. 23 that correspond to values of $0 \leq \mathrm{K}_{\mathrm{v}}$ $\leq \infty$ where $K_{v}=0$ is equivalent to $N_{x y}=0$, and $K_{v}=\infty$ is equivalent to $v=0$ on the loaded edges of a panel. The solid curve corresponds to measured experimental results and the dashed curves correspond to the predicted results. Buckling points are marked by filled squares and circles for the measured and predicted response curves, respectively. The values of the axial load $P$ and the endshortening $\Delta$ are normalized by the corresponding bifurcation buckling load $\mathrm{P}_{\mathrm{cr}}^{\mathrm{o}}$ of a geometrically perfect panel with $v(0, y)=v(L, y)=0$, and the nominal panel thickness $t$, respectively.

The results in Fig. 23 indicate that a linear-elastic circumferential edge restraint has a significant effect on the response of a 60 -in-radius compression-loaded curved panel. For a value of $K_{v}=0\left(N_{x y}(0, y)=N_{x y}(L\right.$, $y)=0$ ), the prebuckling load-shortening response curve is linear and the panel exhibits a buckling point at $\mathrm{P} / \mathrm{P}^{\circ}$ or $=0.87$. For a value of $K_{v^{\prime}}=1.0 \times 10^{5} \mathrm{lb} / \mathrm{in}$., the load-shortening behavior is significantly different. For this case, the panel exhibits a significant amount of prebuckling nonlinearity and has a relatively high buckling load of $P /$ $\mathrm{P}_{\mathrm{cr}}^{\mathrm{r}}=1.93$. This increase in the nonlinearity of the prebuckling load-shortening response is caused by an increase in the out-of-plane prebuckling deformations of the panel which result in a corresponding decrease in the 
instantaneous effective axial stiffness of the panel, but with an overall stiffening effect. with respect to the buckling resistance. The results show that the out-of-plane prebuckling deformations retard the onset of buckling and results in about a $30 \%$ increase in the buckling load with respect to the predicted buckling load for the panel with $v\left(0, y^{\prime}\right)=v($ L. $y)=0$ on the loaded boundary. For values of $\mathrm{K}_{\mathrm{v}}=1.0 \times 10^{7} \mathrm{lb} / \mathrm{in}$. and $\infty$, the panel exhibits less nonlinearity in its load-shortening response curves and the buckling loads decrease to $\mathrm{P} / \mathrm{P}^{\circ}{ }_{\mathrm{cr}}=1.56$ and 1.44 , respectively, as compared to the results for the panel with $\mathrm{K}_{\mathrm{v}}=1.0 \times 10^{5} \mathrm{lb} / \mathrm{in}$. It is important to note that the magnitude of the buckling load and the severity of the nonlinear prebuckling deformations are not bounded by the results for $\mathrm{K}_{\mathrm{v}}=0$ and $\mathrm{K}_{\mathrm{v}}=\infty$, which is contrary to intuition. In addition, the measured buckling load lies between the predicted buckling loads for the panels with $\mathrm{K}_{\mathrm{v}}=1.0 \times 10^{5} \mathrm{lb} / \mathrm{in}$. and $1.0 \times 10^{7} \mathrm{lb} / \mathrm{in}$.

The effects of elastic circumferential edge restraints on the transverse center displacement $\delta$ are illustrated in Fig. 24 where $\delta=w(L / 2, W / 2)$. Five curves are shown in this figure that correspond to values of $0 \leq$ $\mathrm{K}_{\mathrm{v}} \leq \infty$. The solid curves correspond to measured experimental results and the dashed curves correspond to the predicted results. Buckling points are marked by filled squares and circles for the measured and predicted response curves, respectively.

For a value of $\mathrm{K}_{\mathrm{v}}=0$, the prebuckling center displacement is small and inward. Near the buckling load, and in the postbuckling range of loading, the magnitude of the inward displacement grow's rapidly to many times the thickness of the panel. For values of $K_{v}=1.0 \times 10^{5} \mathrm{lb} /$ in., $1.0 \times 10^{7} \mathrm{lb} / \mathrm{in}$, and $\infty$, the center displacement response is characterized by a monotonically increasing outward displacement. This result is consistent with the increase in the degree of nonlinearity of the prebuckling load-shortening response. The results also indicate that the experimentally measured center displacement response is initially bounded by the results for $K_{v}=$ $1.0 \times 10^{5} \mathrm{lb} / \mathrm{in}$. and $\mathrm{K}_{v}=1.0 \times 10^{7} \mathrm{lb} / \mathrm{in}$., but as the loading increases in the prebuckling range, the measured results gradually move out of the bounds of the predicted results.

The effects of elastic circumferential edge restraints on the predicted circumferential (v) displacement of one corner of a 60 -in-radius panel is illustrated in Fig. 25. where $v_{0}=v(0,0)$. Three curves are shown in Fig. 25 that correspond to values of $0 \leq K_{v} \leq \infty$. Buckling points are marked by filled squares. The values of circumferential displacement $v_{0}$ are normalized by the panel thickness 1. The vertical dashed line corresponds to $v_{0}=0$ and is included in this figure for clarity.
For a value of $K_{v}=0$, the corner of the panel expands continuously in the negative $y$-circumferential direction throughout the compression response. For a value of $\mathrm{K}_{\mathrm{V}}=1.0 \times 10^{7} \mathrm{lb} / \mathrm{in}$., the corner of the panel expands circumferentially throughout the compression response, but to a much lesser degree than that seen for the panel with $K_{v}=0$. For a value of $K_{v}=1.0 \times 10^{5} \mathrm{lb} / \mathrm{in}$., however, the corner initially expands circumferentially. but then, upon further loading, begins to contract circumferentially. This reversal in the circumferential displacement takes place at a value of $\mathrm{P} / \mathrm{P}^{\circ}{ }_{\mathrm{cr}}=0.94$ which corresponds closely with the load level at which the loadshortening response curve transitions from a linear to a nonlinear shape (see Fig. 23) and the panel exhibits a rapid growth in the magnitude of transverse center displacement, as indicated in Fig. 24.

Predicted displacement contours for panels at the onset of buckling with various values of $\mathrm{K}_{\mathrm{v}}$ are presented in Fig. 26. The dashed contour lines in Figs. 26(b) through $26(\mathrm{e})$ represent inward displacements and the solid lines represent outward displacements. The density of the contour lines is indicative of the severity of the displacement gradients in the panel. In addition, the buckling load values are included in the figure for each of the panels.

These results in Fig. 26 indicate that the displacement response changes significantly as the circumferential boundary stiffnesses $\mathrm{K}_{\mathrm{V}}$ increases. For a value of $\mathrm{K}_{\mathrm{V}}$ $=0$, the panel displacements are characterized by a large centrally located inward deformation pattern, as shown in Fig. 26b. For values of $\mathrm{K}_{\mathrm{v}}=1.0 \times 10^{7} \mathrm{lb} / \mathrm{in}$. and $\infty$, the predicted panel displacement are characterized by a large centrally located outward deformation pattern as shown in Figs. 26d and 26e, respectively. For a value of $\mathrm{K}_{\mathrm{V}}=$ $1.0 \times 10^{5} \mathrm{lb} / \mathrm{in}$., however, the predicted displacement contours in Fig 26c are characterized by an hour-glass pattern similar to the observed moirè fringe pattern shown in Fig. 26a.

A summary of the predicted effects of elastic circumferential edge restraints on the magnitude of the buckling load of a 60 -in-radius, geometrically perfect panel is presented in Fig 27. Predicted buckling load values are marked in the figure by filled square symbols and connected with solid line segments.

The predicted results in Fig. 27 can be placed into three distinct groups. The first group of results are for panels with $0 \leq \mathrm{K}_{\mathrm{V}} \leq 3.0 \times 10^{4}$. These panels exhibit a linear prebuckling load-shortening response similar to the predicted response for $\mathrm{K}_{\mathrm{v}}=0$ that is shown in Fig 23 . The buckling load values for these panels are all below the predicted linear bifurcation buckling load. In addition, these pancls exhibit transverse center displacement responses, circumferential corner displacement respons- 
es and displacement contours similar to the predicted response for $\mathrm{K}_{\mathrm{v}}=0\left(\mathrm{~N}_{\mathrm{xy}}=0\right)$ shown in Fig 24, 25 and $26 \mathrm{~b}$. respectively. The second group of results are for panels with $4.0 \times 10^{4} \leq \mathrm{K}_{\mathrm{v}} \leq 3.0 \times 10^{6}$. The panels in this group exhibit a significant nonlinear prebuckling response similar to the predicted response discussed previously herein for a panel with $\mathrm{K}_{\mathrm{v}}=1.0 \times 10^{5} \mathrm{lb} / \mathrm{in}$. The prebuckling load-shortening response for this group of panels exhibits a significant amount of nonlinearity, and the buckling load values can exceed twice the predicted linear bifurcation buckling load value. In addition, the character of the displacement response is similar to that predicted for the $\mathrm{K}_{\mathrm{r}}=1.0 \times 10^{5} \mathrm{lb} / \mathrm{in}$. case that is illustrated in Figs. 24 , 25 , and $26 \mathrm{c}$. The final group of results are for panels with $4.0 \times 10^{6} \leq \mathrm{K}_{\mathrm{v}} \leq \infty$. The panels associated with this group exhibit response characteristics similar to those predicted for the fully clamped, $\mathrm{K}_{\mathrm{v}}=\infty(\mathrm{v}=0)$ case, that is illustrated in Figs. 23, 24, 25 and 26e.

\section{Concluding Remarks}

Results from a numerical and experimental study of compression-loaded quasi-isotropic curved panels with a centrally located circular cutout have been presented. The numerical results were obtained by using a geometrically nonlinear finite-element analysis code. Some of the effects of cutout size, panel curvature, initial geometric imperfections, and elastic circumferential edge restraints on the prebuckling, buckling and postbuckling the response have been described. These results indicate that a cutout can have a significant effect on the prebuckling, buckling and postbuckling response of a compression-loaded panel. For example, the results indicate that an increase in the cutout size results in a decrease in the effective prebuckling stiffness of the panel, as expected, but causes an increase in the nonlinearity of the loadshortening response curve. For relatively small values of the cutout-diameter-to-panel-width ratio $\mathrm{d} / \mathrm{W}$, the panels exhibit a distinct unstable buckling event. For relatively large values of $\mathrm{d} / \mathrm{W}$, the panel response no longer exhibits an unstable buckling event; rather, the panel response is characterized by a monotonically increasing loadshortening curve. The results also show that the value of $\mathrm{d} / \mathrm{W}$ that marks the transition between these two types of nonlinear behavior depends significantly on the panel curvature. In particular, panels with a 15 -in-radii exhibit the transition at a value of $\mathrm{d} / \mathrm{W} 0.4<\mathrm{d} / \mathrm{W}<0.6$, and the 60 -in-radius panels exhibit the transition between $\mathrm{d} / \mathrm{W}$ $0.2<\mathrm{d} / \mathrm{W}<0.3$. Results have been presented that also show that the buckling loads for panels with small values of $\mathrm{d} / \mathrm{W}$ are more sensitive to initial geometric imperfections than panels with large values of $d / W$. This trend is consistent with the idea that, as the amount of material in the center of the panel decreases and the prebuckling de- formations near the cutout dominate the response. the panels become less sensitive to initial geometric imperfections. When the load-shortening curve is monotonically increasing and there is no buckling event, there also is no imperfection sensitivity.

Correlation between the numerically predicted and experimentally measured results was good in most cases. However, significant discrepancies and unexpected behavioral characteristics were obtained for certain cases. For example, the shallow 60-in-radius panels with small values of $\mathrm{d} / \mathrm{W}$ exhibit a significant amount of nonlinearity in the prebuckling portion of the load-shortening response curve and exhibit buckling load values that are significantly greater than the predicted linear bifurcation buckling load for a 60-in-radius geometrically perfect panel without a cutout. This behavior is contrary to previously known behavioral characteristics of compression-loaded shallow curved panels. Predicted and observed buckling deformations for 15 - and 60-in-radius panels with $\mathrm{d} / \mathrm{W}=0$ do not correlate well. Furthermore, predicted buckling load values for 15 - and 60 -in-radius panels with small values of $d / W$ are consistently underpredicted when compared to the experimentally measured buckling load values. The largest discrepancy between measured and predicted buckling load occurs for a 60 -in-radius panel with $\mathrm{d} / \mathrm{W}=0$, in which case the predicted buckling load value is $30 \%$ less than the measured value. However, the observed discrepancy between the measured and predicted buckling loads decreases as the value of $d / W$ increases.

In an effort to explain some of the behavioral issues stated above, results from a limited numerical parametric study of the effects of elastic circumferential edge restraints on the compression response of a 60 -in-radius geometrically perfect panel have been presented. These results indicate that an elastic circumferential edge restraint on the loaded edges of a curved panel has a significant effect on the behavioral characteristics. Overall, the study has shown that the predicted results can be placed in to three groups that exhibit distinct behavioral characteristics depending on the magnitude of the elastic circumferential restraint. Panels in the first group with a relatively low magnitude, approximately $3 \%$ of the panels circumferential membrane stiffness $\mathrm{A}_{22}$. exhibit a linear load-shortening response that contains a distinct buckling point. The magnitude of the buckling load for these panels is always less than the predicted linear bifurcation buckling load and the prebuckling deformations are negligible. Panels in the second group have an intermediate magnitude of the elastic circumferential restraint, $4 \%$ of $\mathrm{A}_{22} 103.25 \mathrm{~A}_{22}$, exhibit a significant amount of nonlinearity in the prebuckling response and can exhibit buckling loalds that are more than twice the predicted linear bifurcition buckling load. Panels in the third group are for relatively high magnitudes of circum- 
ferential restraint. greater than $3.25 \mathrm{~A}_{22}$. exhibit some nonlinearity but not nearly as pronounced as the nonlinear behavior exhibited by the second group of panels. The predicted results for the panels with an intermediate magnitude of the elastic circumferential restraint more closely represent the experimentally measured results and indicate that some amount of restricted circumferential motion at the loaded boundary is present in the test fixture during the experiments.

\section{Acknowledgments}

The work presented herein was supported by the Structural Mechanics Branch, NASA Langley Research Center and the National Research Council, Grant Number NASW-8154.

\section{$\underline{\text { References }}$}

${ }^{1}$ Nemeth, M. P., "Buckling and Postbuckling Behavior of Laminated Composite Plates With a Cutout," NASA TP-3587, July 1996.

${ }^{2}$ Nemeth, M. P., "Buckling and Postbuckling Behavior of Square Compression-Loaded Graphite-Epoxy Plates With Circular Cutouts," NASA TP-3007, 1990.

${ }^{3}$ Nemeth, M. P., "Buckling Behavior of CompressionLoaded Symmetrically Laminated Angle-Ply Plates With Holes," AIAA J., Vol. 26, No. 3, March 1988, pp. 330-336.

${ }^{4}$ Nemeth, M. P. and Starnes, J. H., Jr., "The NASA Monographs on Shell Stability Design Recommendations: A Review and Suggested Improvements," NASA TP-1998-206290, January 1998.

${ }^{5}$ Madenci, E. and Barut, A.. "Pre- and Postbuckling Response of Curved, Thin, Composite Panels With Cutouts Under Compression," International Journal for Numerical Methods in Engineering, Vol. 37, 1994, pp.1499-1510.

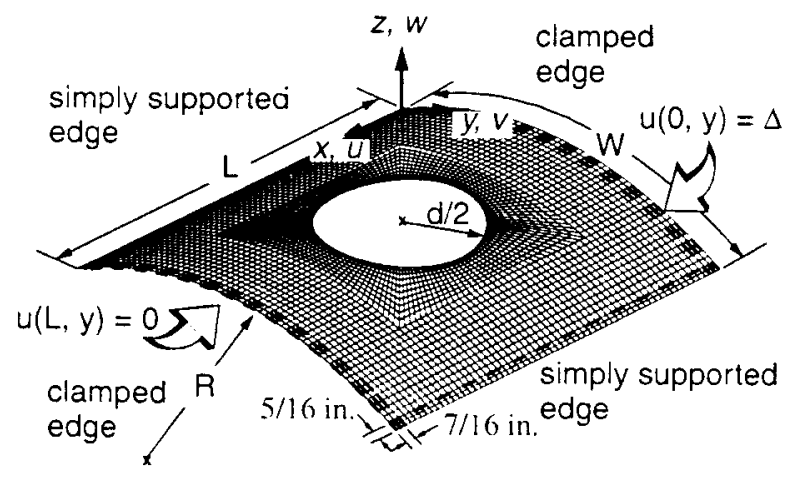

Fig. 1 Typical model geometry and boundary conditions (dashed lines mark the rows on which the boundary conditions are applied).
${ }^{6}$ Knight, N. F. and Starnes. J. H., Jr., "Postbuckling Behavior of Axially Compressed Graphite-Epoxy' Cylindrical Panels with Circular Holes." Proceedings of the 1984 ASME Joint Pressure Vessels and Piping/ Applied Mechanics Conference. 1984.

7Janisse, T. C. and Palazotto, A. N.. "Collapse Analysis of Composite Panels With Cutouts," Proceedings of the AIAA/ASME/ASCE/AHS 24th Structures, Structural Dynamics, and Materials Conference, AIAA paper 83$0875,1983$.

${ }^{8}$ Lee, C. E. and Palazotto, A. N., “Nonlinear Collapse Analysis of Composite Cylindrical Panels With Small Cutouts or Notches," Proceedings of the AIAA/ASME/ ASCE/AHS 25th Structures, Structural Dynamics, and Materials Conference, AIAA paper 84-0889, 1984.

${ }^{9}$ Brogan, F. A., Rankin, C. C., and Cabiness, H. D., "STAGS Users Manual," Lockheed Palo Alto Research Laboratory, Report LMSC P032594, 1994.

${ }^{10}$ Nemeth, M. P., "Nondimensional Parameters and Equations for Buckling of Anisotropic Shallow Shells," J. of Applied Mechanics, Vol. 61, September 1994, pp. 664-669.

${ }^{11}$ Knight, N. F. and Starnes, J. H., Jr., "Postbuckling Behavior of Selected Graphite-Epoxy Cylindrical Panel Loaded in Compression," Proceedings of the AIAA/ ASME/ASCE/AHS 27th Structures, Structural Dynamics, and Materials Conference, AIAA paper 86-0881CP, May 1986.

${ }^{12}$ Snell, M. B. and Morley, N. T., "The Compression Buckling Behaviour of Highly Curved Panels of Carbon Fibre Reinforced Plastic, "Proceedings of the Fifth International Conference on Composite Materials, ICCM Vol. 5, August 1985, pp. 1327-1354.

${ }^{13}$ Wilkins, D. J., "Compression Buckling tests of Laminated Graphite-Epoxy Curved Panels," AIAA J., Vol. 13, No. 4 April 1975, pp. 465-470.

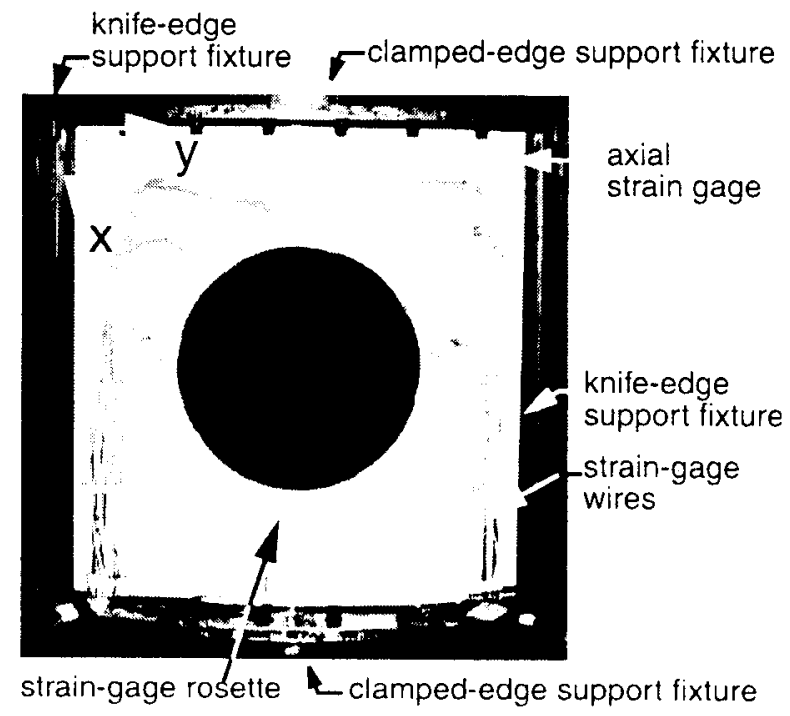

Fig. 2 Typical test specimen and apparatus. 


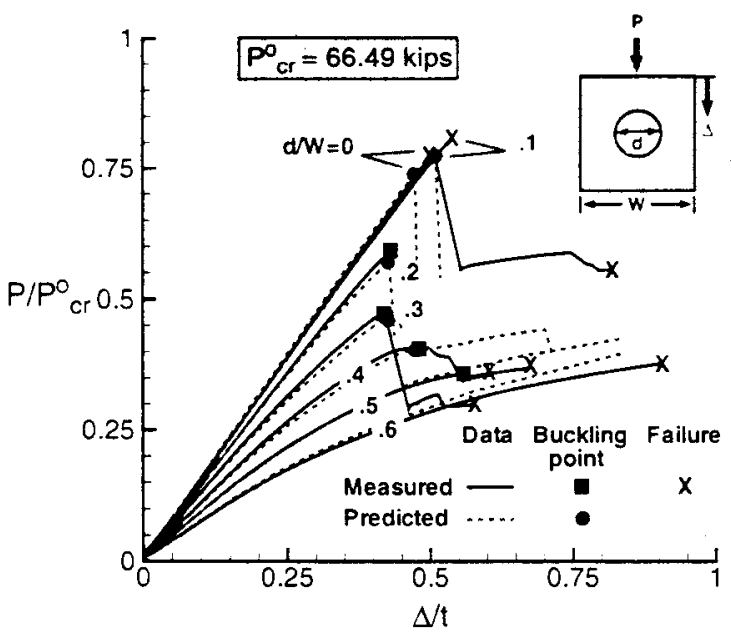

Fig. 3 Numerically predicted and experimentally measured load-shortening response curves for 15-in-radius panels with a cutout

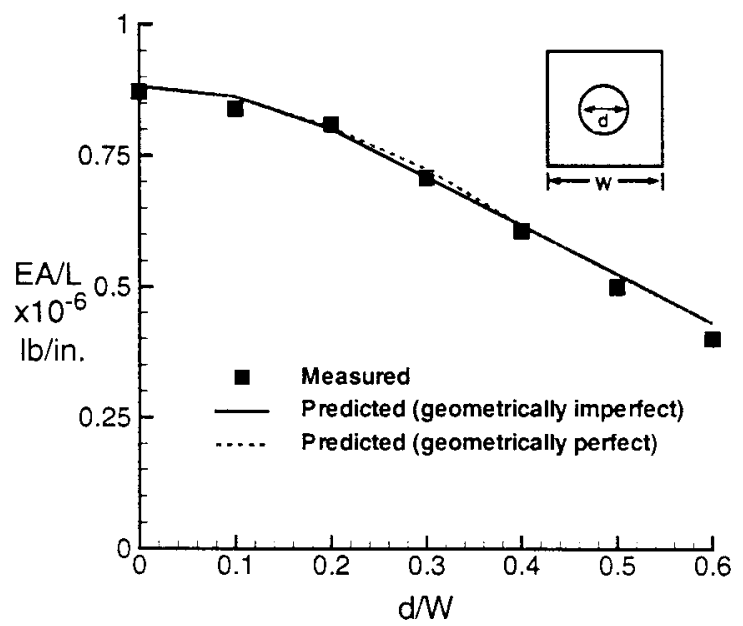

Fig. 4 Numerically predicted and experimentally measured effective prebuckling stiffness for 15 -in-radius panels with a cutout.

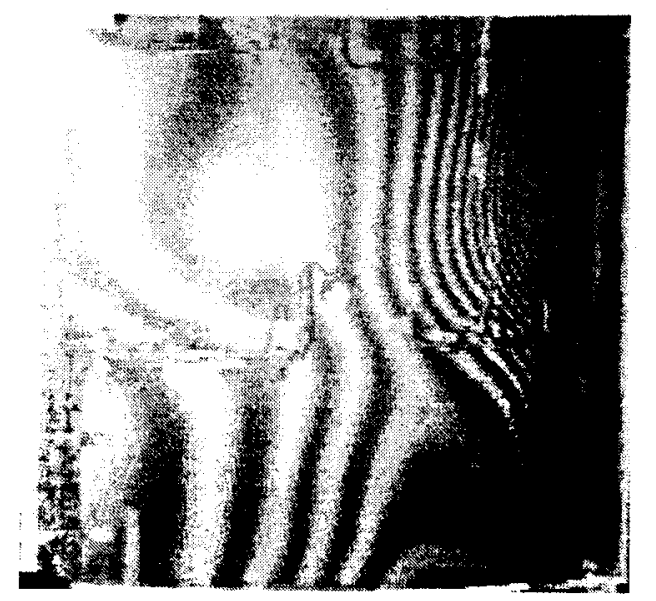

(a) Moirè fringe pattern at the onset of buckling, $\mathrm{P} / \mathrm{P}^{(}{ }_{\text {cr }}=.77$

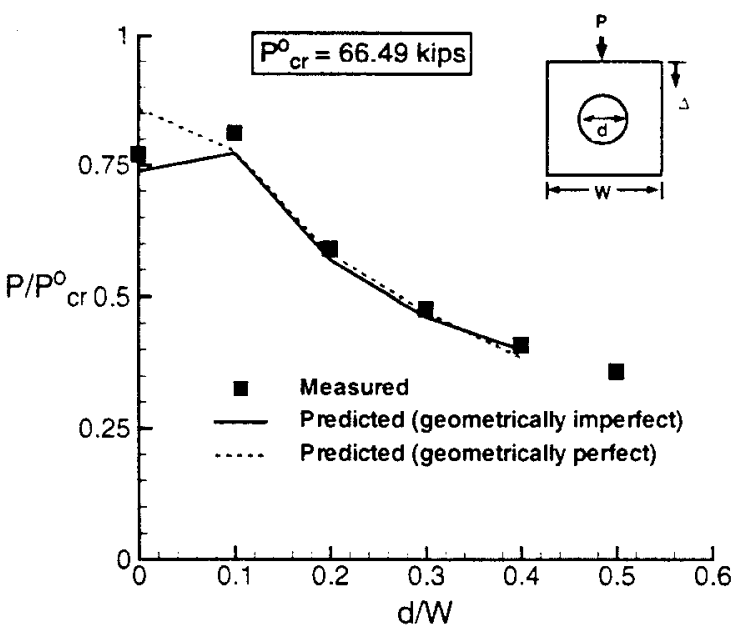

Fig. 5 Numerically predicted and experimentally measured buckling load for 15 -in-radius panels with a cutout.

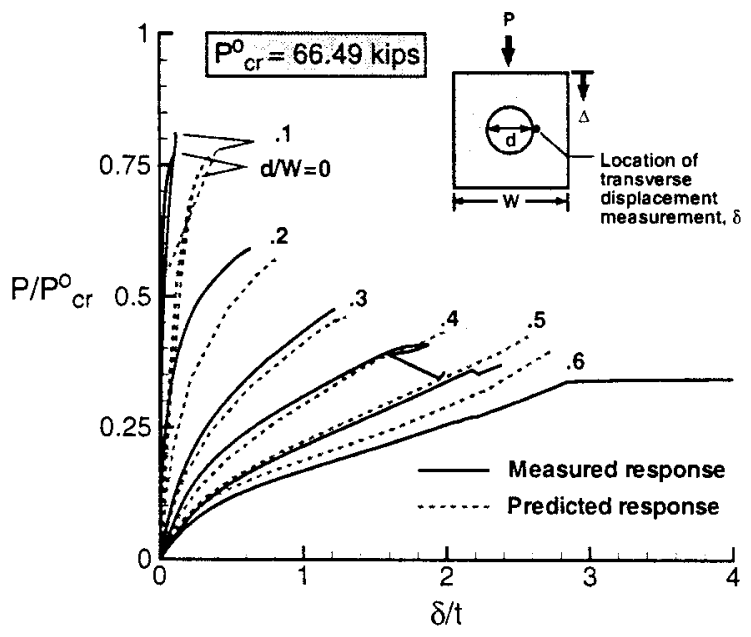

Fig. 6 Numerically predicted and experimentally measured out-of-plane center displacements for 15 -in-radius panels with a cutout.

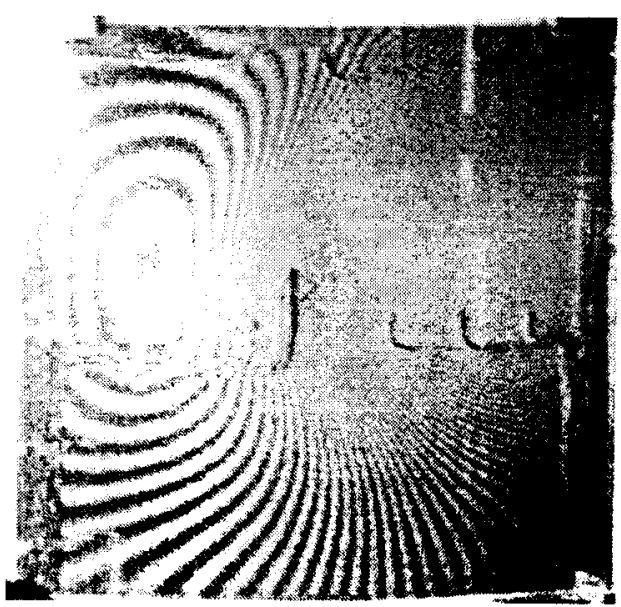

(b) Initial postbuckling moirè fringe pattern, $\mathrm{P} / \mathrm{P}^{\mathrm{s}}{ }_{\mathrm{cr}}=.53$

Fig. 7 Observed and predicted out-of-plane displacements for a 15 -in-radius pancl with $\mathrm{d} / \mathrm{W}=0$. 


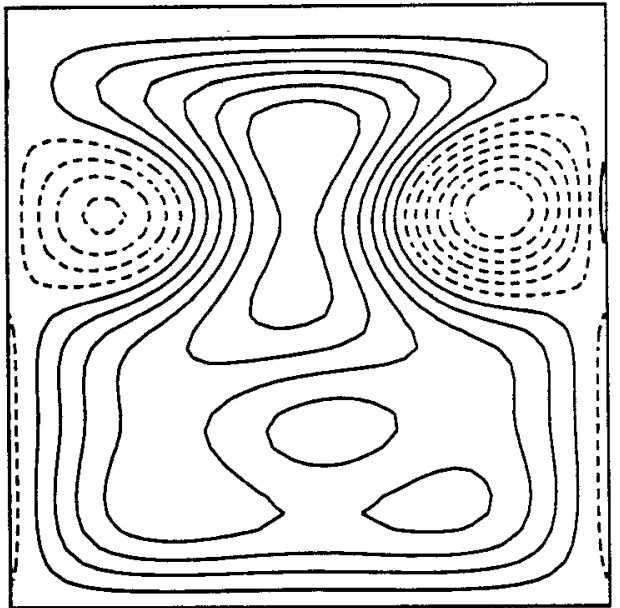

(c) Predicted displacements at the onset of buckling, $\mathrm{P} / \mathrm{P}_{\mathrm{cr}}^{0}=.75$

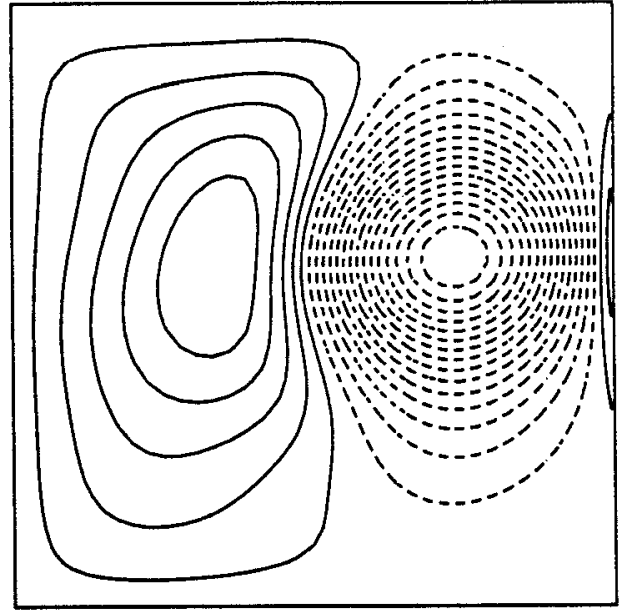

(d) Predicted initial postbuckling displacements, $\mathrm{P} / \mathrm{P}^{\mathrm{o}}{ }_{\mathrm{cr}}=.53$

Fig. 7 Continued.

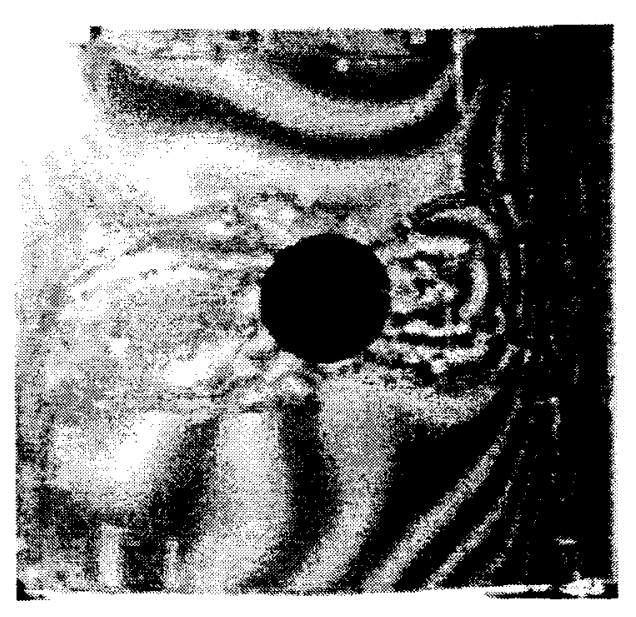

(a) Moirè fringe pattern at the onset of buckling, $\mathrm{P} / \mathrm{P}_{\text {cr }}=.59$

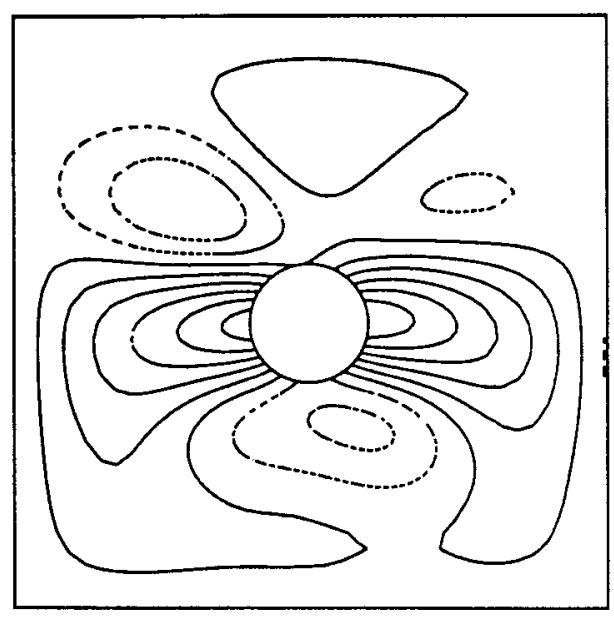

(c) Predicted displacements at the onset of buckling, $\mathrm{P} / \mathrm{P}^{\circ} \mathrm{cr}=.57$

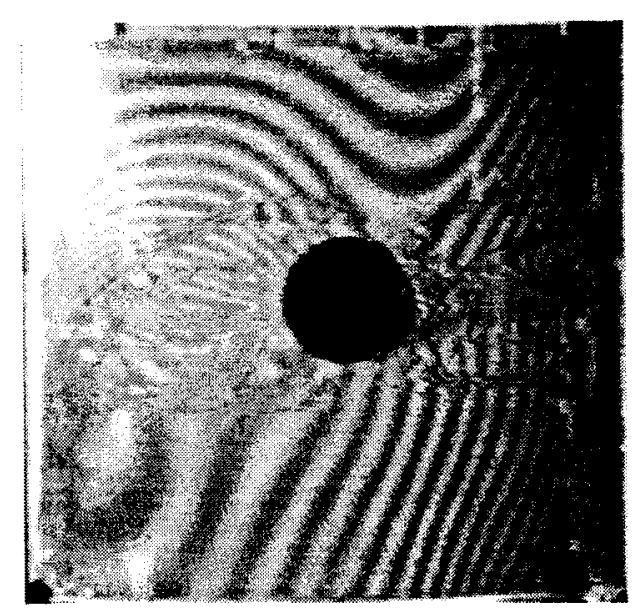

(b) Initial postbuckling moirè fringe pattern, $\mathrm{P} / \mathrm{P}_{\mathrm{cr}}^{\mathrm{O}}=\mathbf{0}$ (failure)

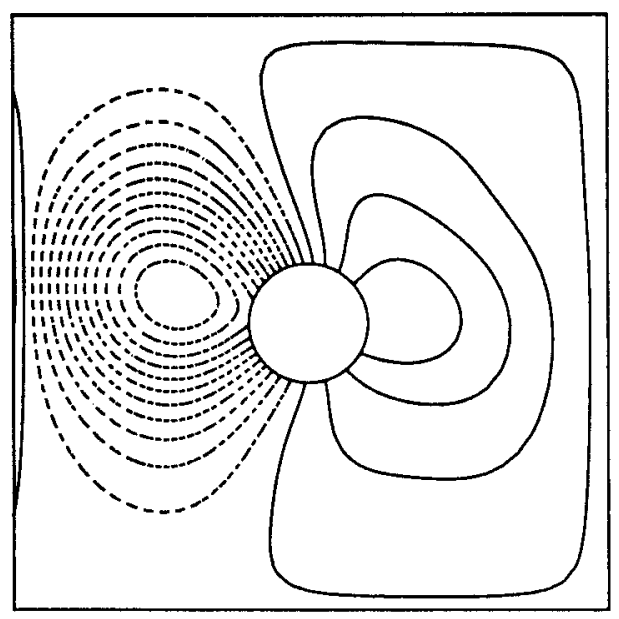

(d) Predicted initial postbuckling displacements, $P / P^{\prime \prime}{ }=.43$

Fig. 8 Observed and predicted out-of-plane displacements for a 15 -in-radius pancl with $\mathrm{d} / \mathrm{W}=0.2$. 


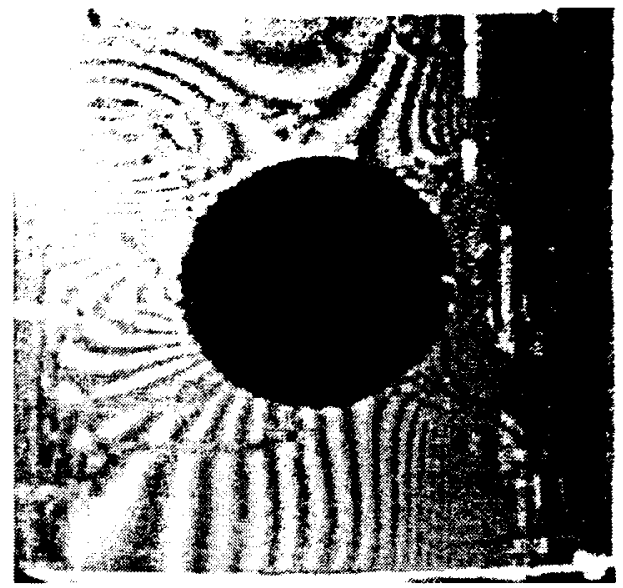

(a) Moirè fringe pattern at the onset of buckling, $\mathrm{P} / \mathrm{P}_{\mathrm{cr}}^{\mathrm{c}}=.40$

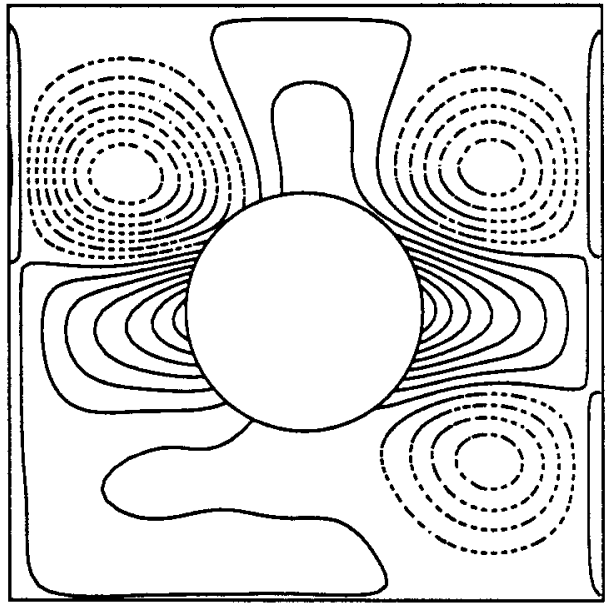

(c) Predicted displacements at the onset of buckling, $\mathrm{P} / \mathrm{P}_{\mathrm{cr}}^{\circ}=.40$

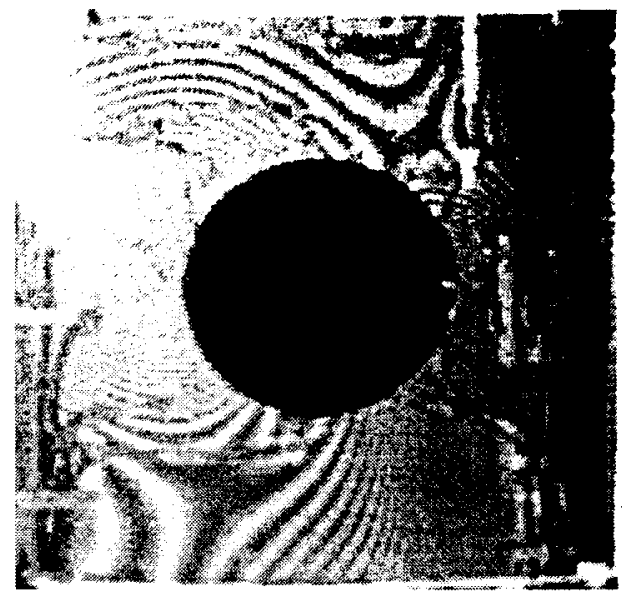

(b) Initial postbuckling moirè fringe pattern, $\mathrm{P} / \mathrm{P}^{0}{ }_{\mathrm{cr}}=.39$

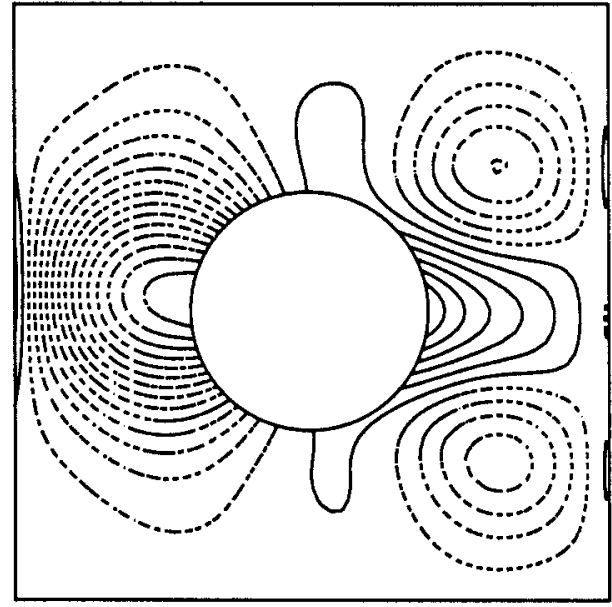

(d) Predicted initial postbuckling displacements, $\mathrm{P} / \mathrm{P}_{\mathrm{cr}}^{\mathrm{o}}=.39$

Fig. 9 Observed and predicted out-of-plane displacements for a 15 -in-radius panel with $\mathrm{d} / \mathrm{W}=0.4$.

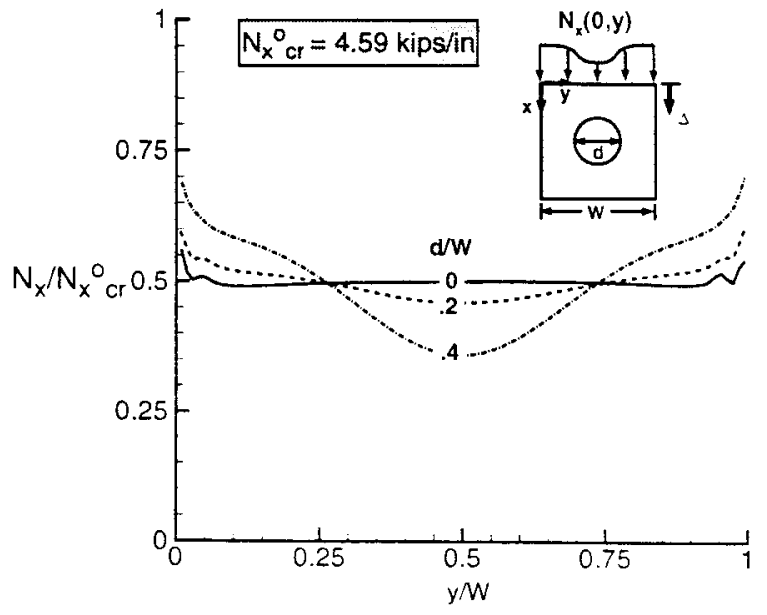

Fig. 10 Stress resultant distributions on the loaded end of a 15 -in-radius panel with a cutout and $P / P^{0}{ }^{\circ}{ }^{2}=0.5$.

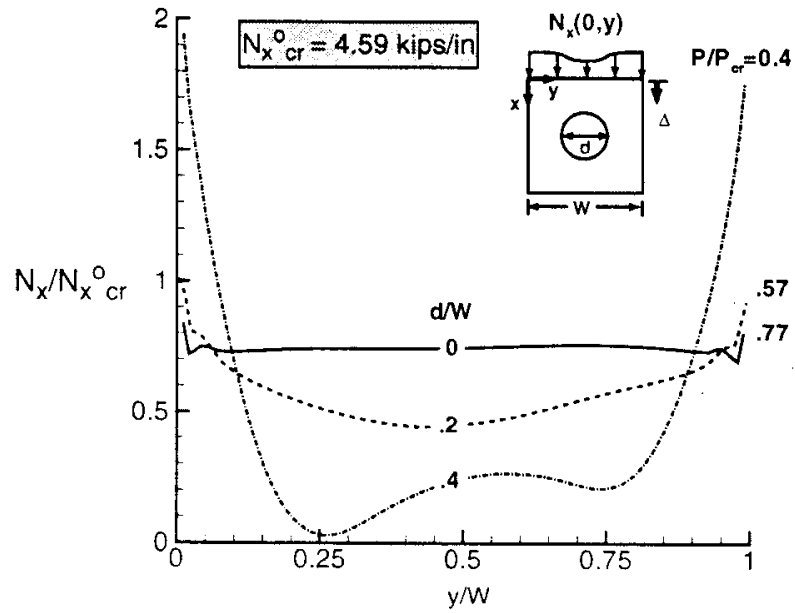

Fig. 11 Stress resultant distribution on the loaded end of a 15-in-radius panel with a cutout at the onset of buckling. 


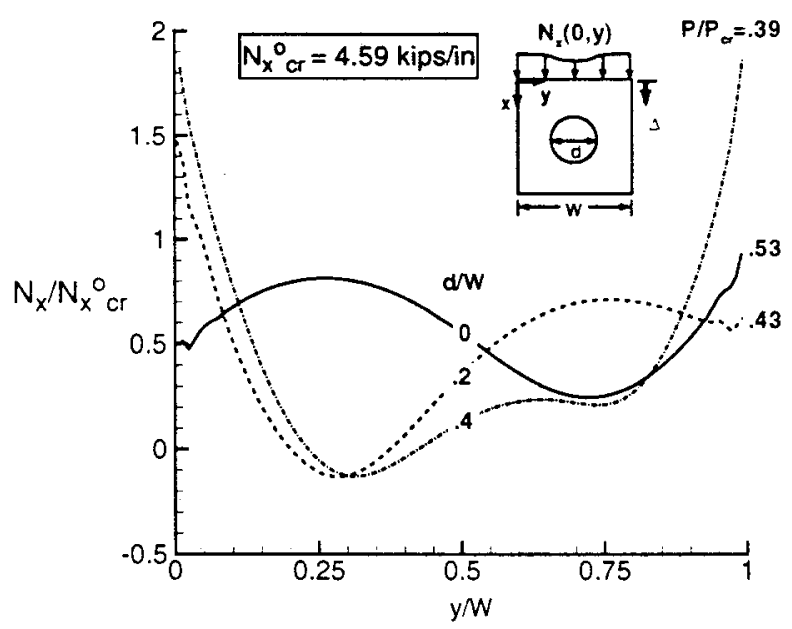

Fig. 12 Initial postbuckling stress resultant distribution on the loaded end of a 15-in-radius panel with a cutout.

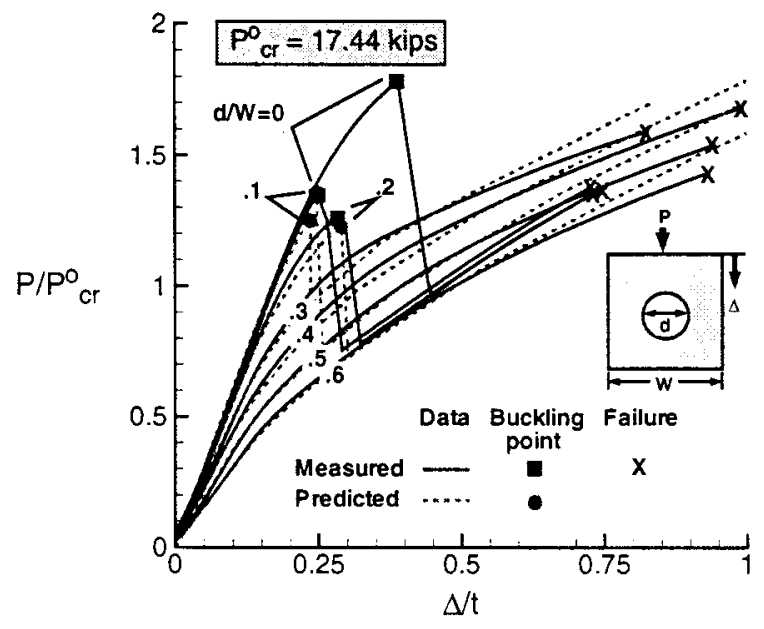

Fig. 13 Numerically predicted and experimentally measured load-shortening response curves for 60 -in-radius panels with a cutout.

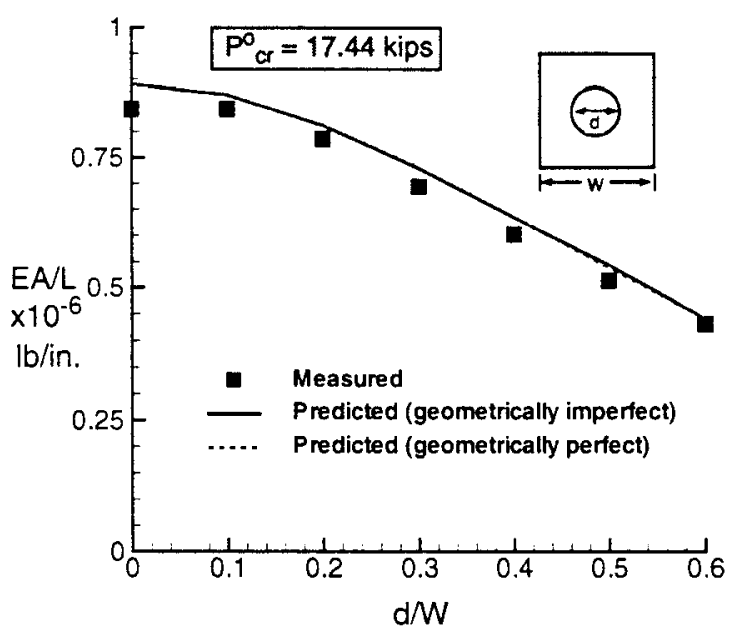

Fig. 14 Numerically predicted and experimentally measured effective initial prebuckling stiffness for 60 -in-radius panels with a cutout.

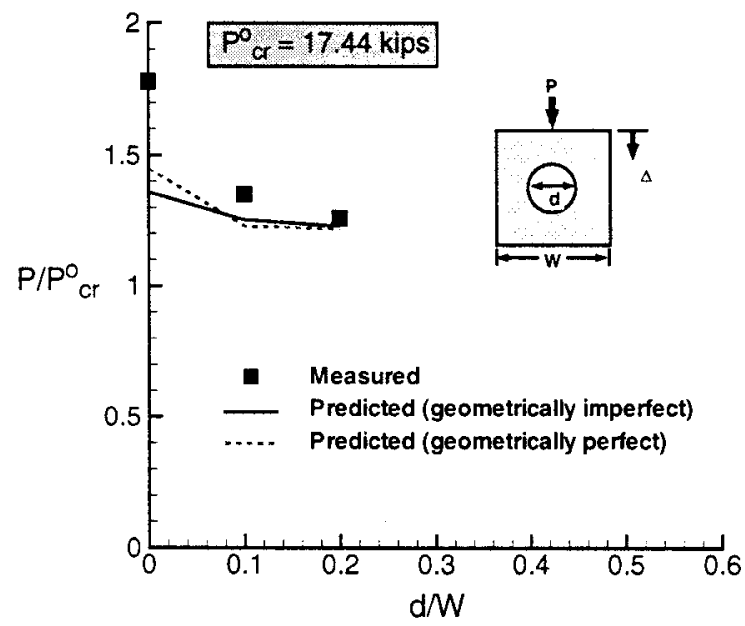

Fig. 15 Numerically predicted and experimentally measured buckling loads for 60 -in-radius panels with a cutout.

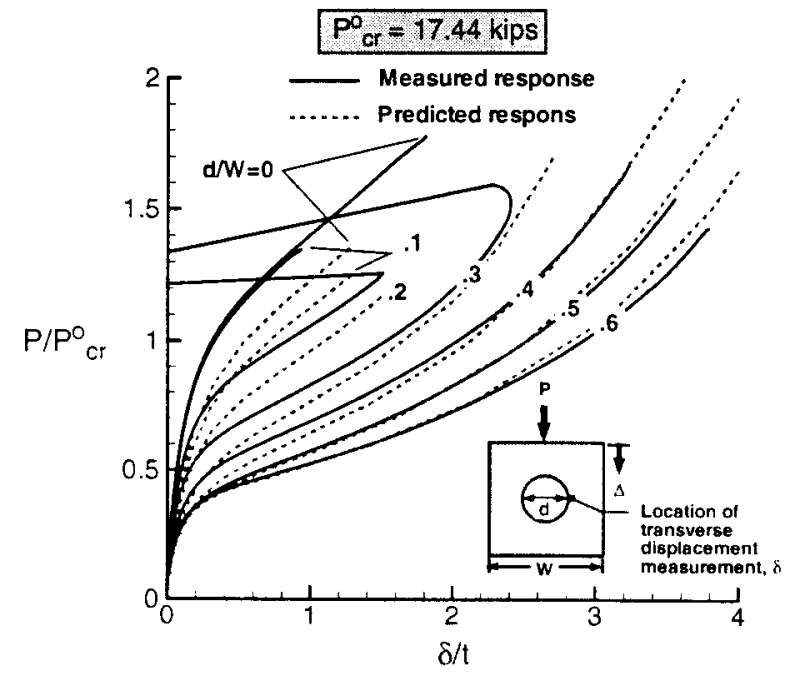

Fig. 16 Numerically predicted and experimentally measured out-of-plane displacements for 15-in-radius panels with a cutout. 


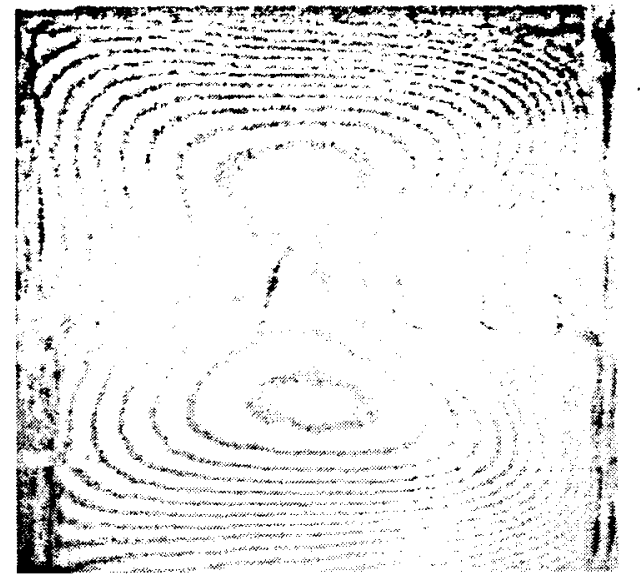

(a) Moirè fringe pattern at the onset of buckling, $\mathrm{P} / \mathrm{P}^{0}{ }_{\mathrm{cr}}=1.75$

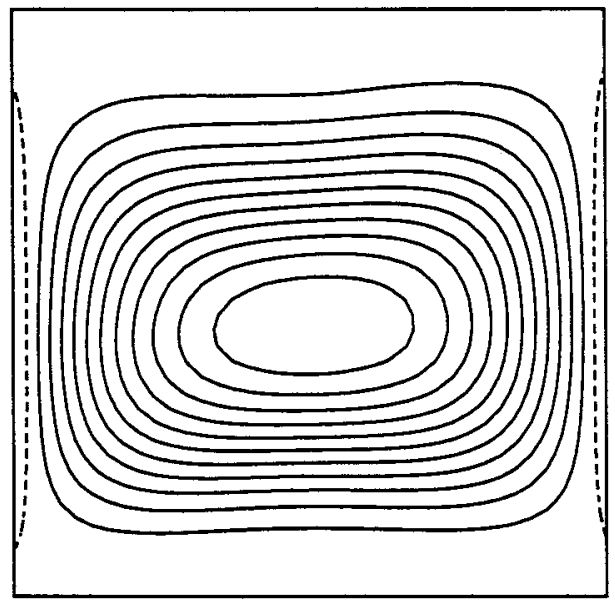

(c) Predicted displacements at the onset of buckling, $\mathrm{P} / \mathrm{P}^{0}{ }_{\mathrm{cr}}=1.35$

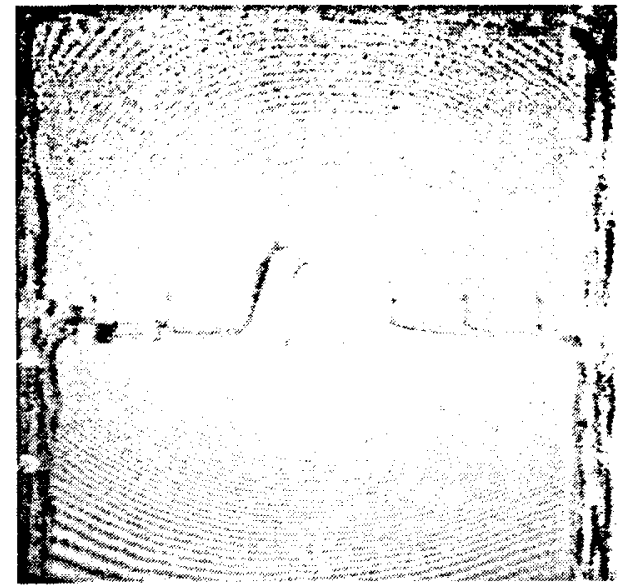

(b) Initial postbuckling moirè fringe pattern, $\mathrm{P} / \mathrm{P}_{\mathrm{cr}}^{0}=.96$

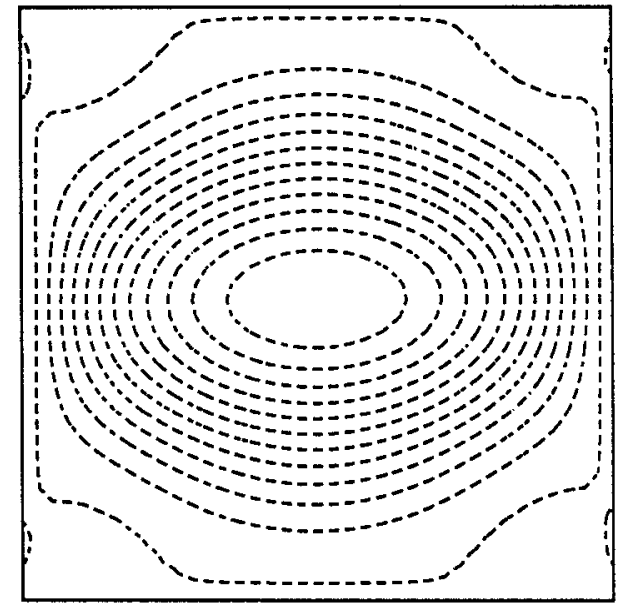

(d) Predicted initial postbuckling displacements, $\mathrm{P} / \mathbf{P}_{\text {cr }}=.73$

Fig. 17 Observed and predicted out-of-plane displacements for a 60 -in-radius panel with $\mathrm{d} / \mathrm{W}=\mathbf{0}$.

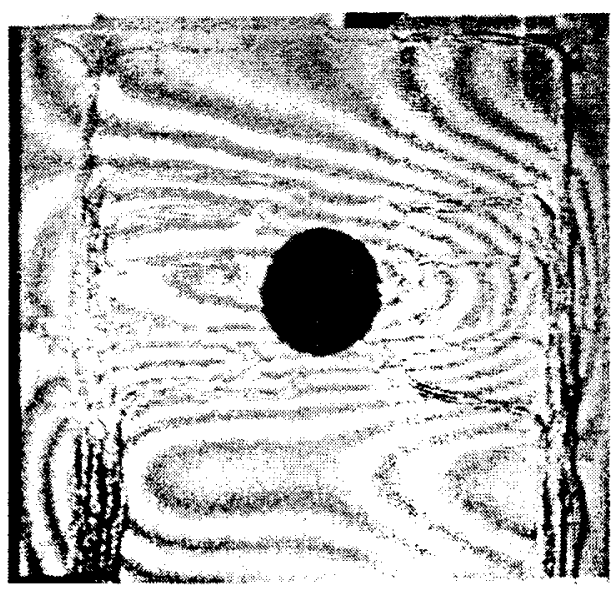

(a) Moire fringe pattern at the onset of buckling, $P / P^{\prime \prime}=1.26$

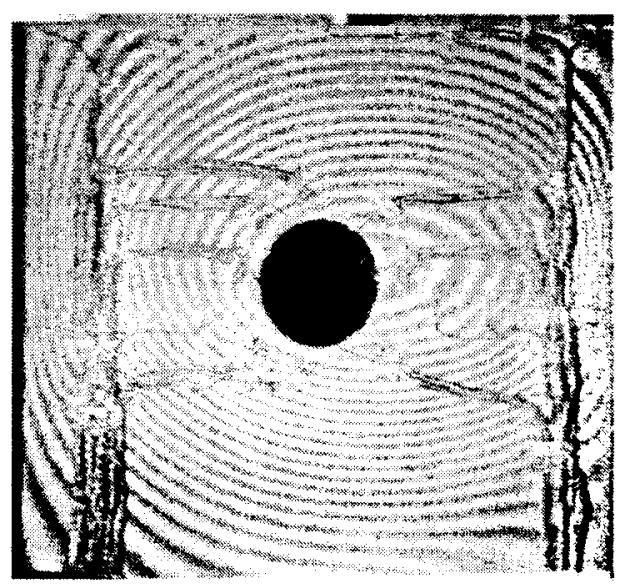

(b) Initial postbuckling moirè fringe pattern. $\mathrm{P} / \mathrm{P}_{\mathrm{cr}}=.75$

Fig. 18 Observed and predicted out-of-plane displacements for a 60-in-radius panel with $\mathrm{d} / \mathrm{W}=0.2$. 


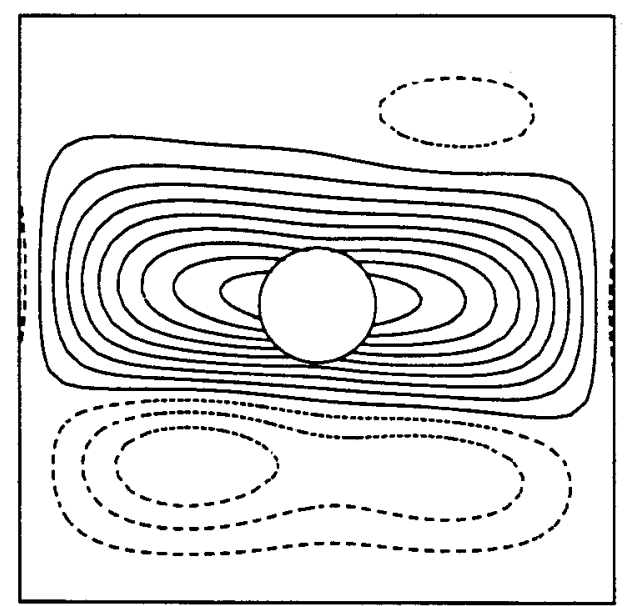

(c) Predicted displacements at the onset of buckling, $\mathrm{P} / \mathrm{P}_{\mathrm{cr}}^{0}=1.23$

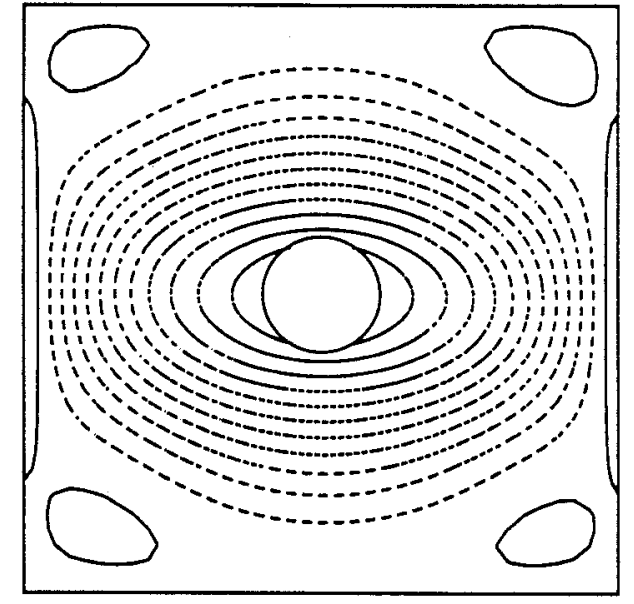

(d) Predicted initial postbuckling displacements, $\mathrm{P} / \mathrm{P}_{\mathrm{cr}}{ }_{\mathrm{cr}}=.74$

Fig. 18 Continued.

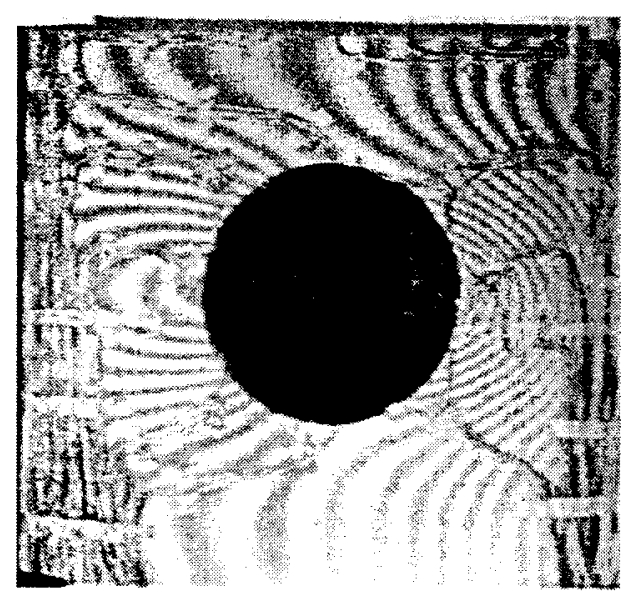

(a) Moirè fringe pattern at $\mathrm{P} / \mathrm{P}^{0}{ }_{\mathrm{cr}}=.63$

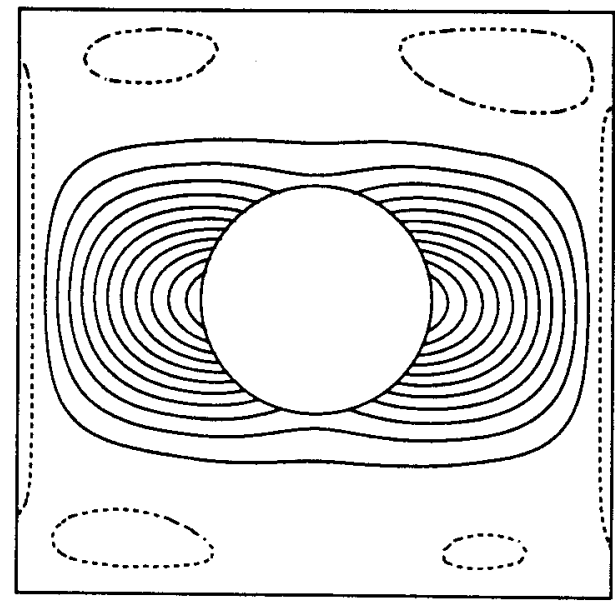

(c) Predicted displacements at $\mathrm{P} / \mathrm{P}^{\prime \prime}{ }^{\prime} \mathrm{r}=.63$

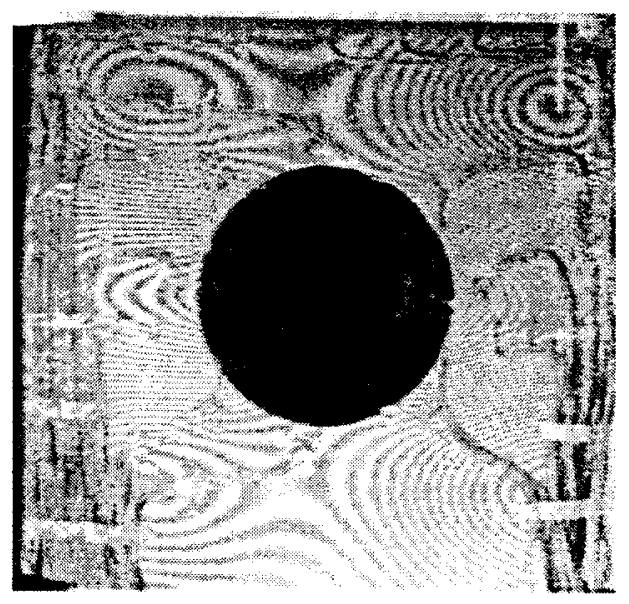

(b) Moirè fringe pattern at $\mathrm{P} / \mathrm{P}^{\mathrm{o}}{ }_{\mathrm{cr}}=1.6$

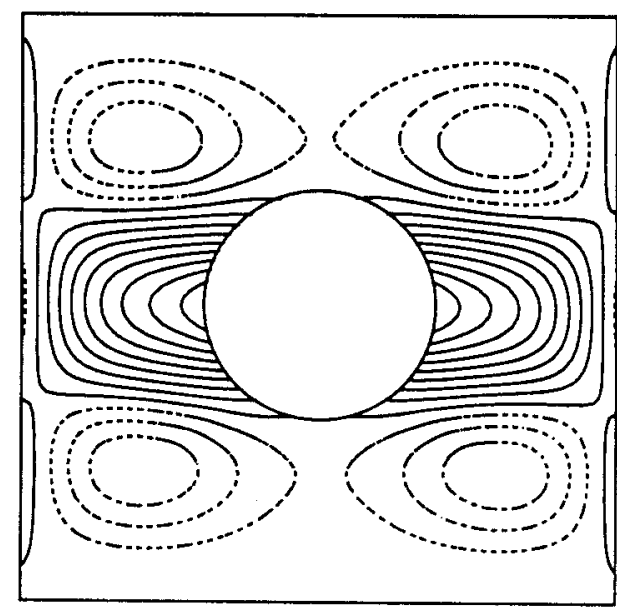

(d) Predicted displacements at $\mathrm{P}^{\mathrm{P}} / \mathrm{P}^{(1)}{ }^{(\mathrm{r}}=1.6$

Fig. 19 Observed and predicted out-of-plane displacements for a 60 -in-radius pancl with $\mathrm{d} / \mathrm{W}=0.4$. 


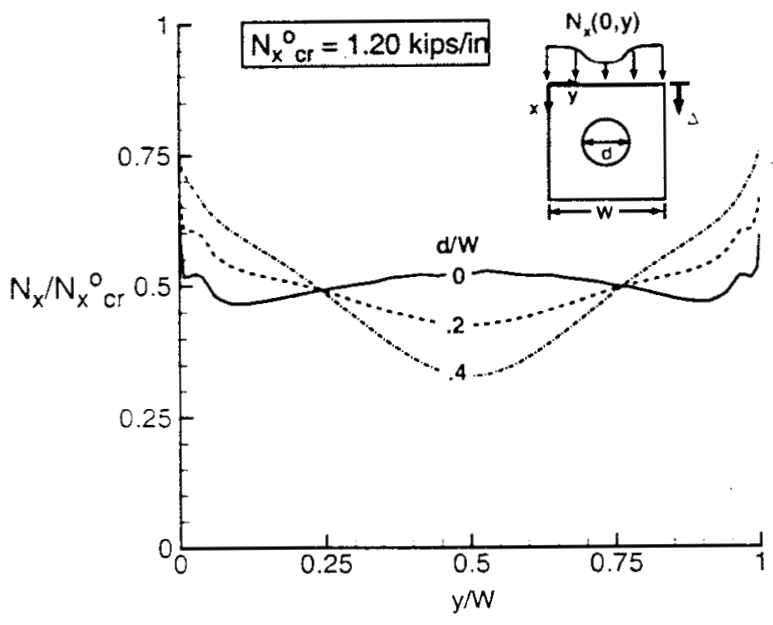

Fig. 20 Stress resultant distributions on the loaded end of a 60 -in-radius panel with a cutout and $P / P^{0}{ }_{c r}=0.5$.

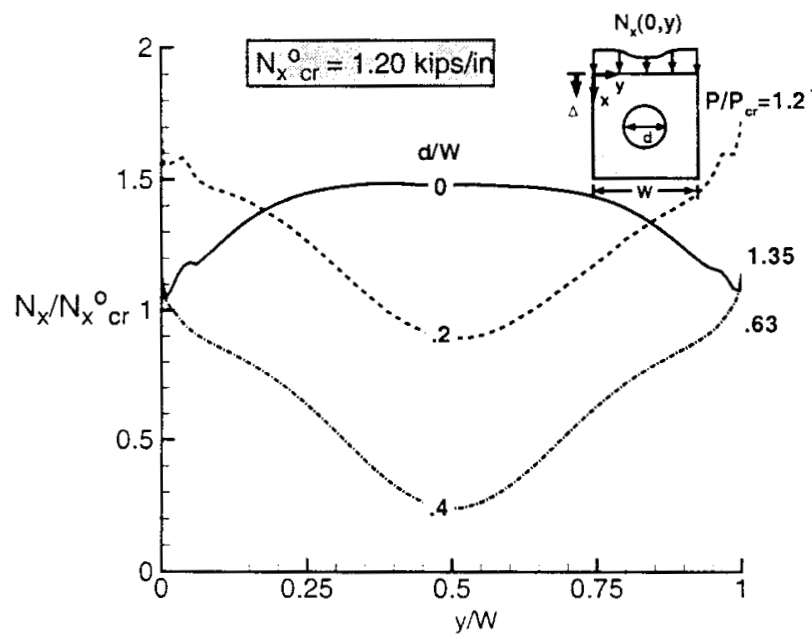

Fig. 21 Stress resultant distributions on the loaded end of a 60-in-radius panel with a cutout at the onset of buckling.

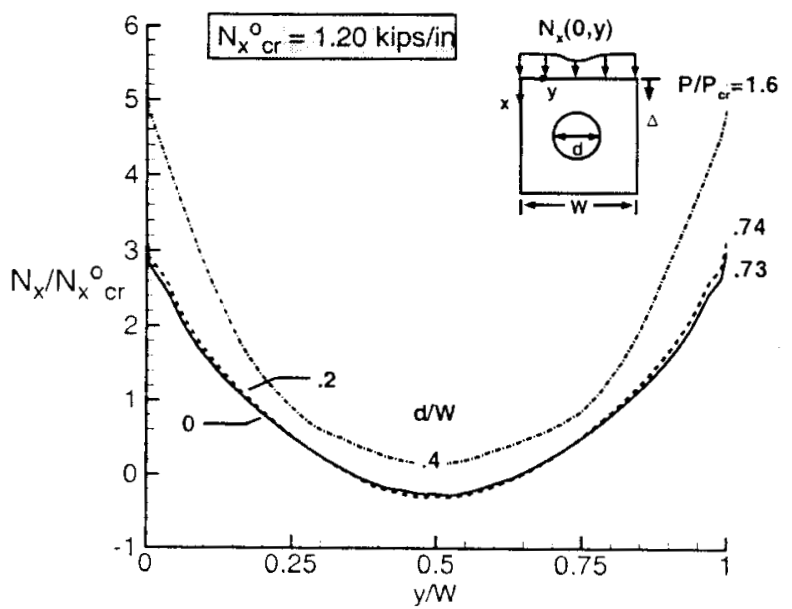

Fï. 22 Initial postbuckling stress resultant distributions on the loaded end of 6(1)-in-radius panelwith a cutout.

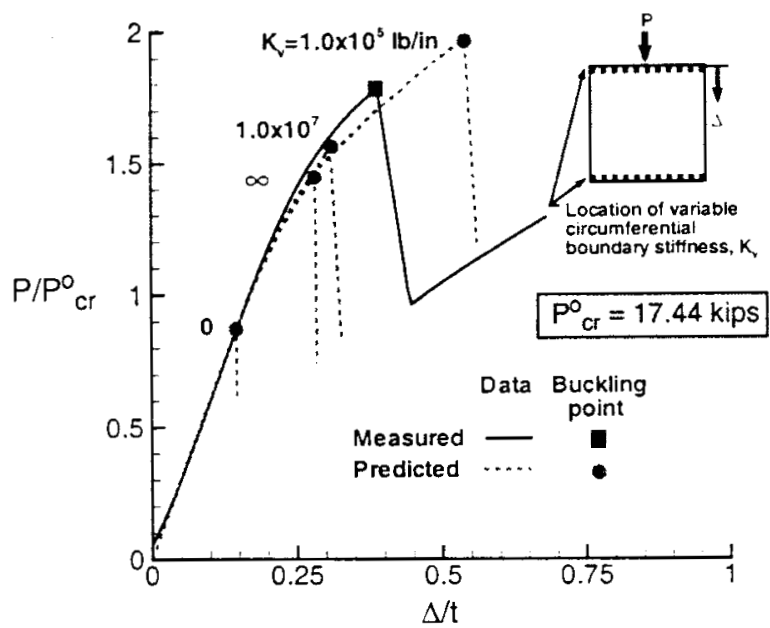

Fig. 23 Effects of circumferential boundary stiffness $K_{v}$ on the load-shortening response of a 60 -in-radius panel with $\mathrm{d} / \mathbf{W}=\mathbf{0}$.

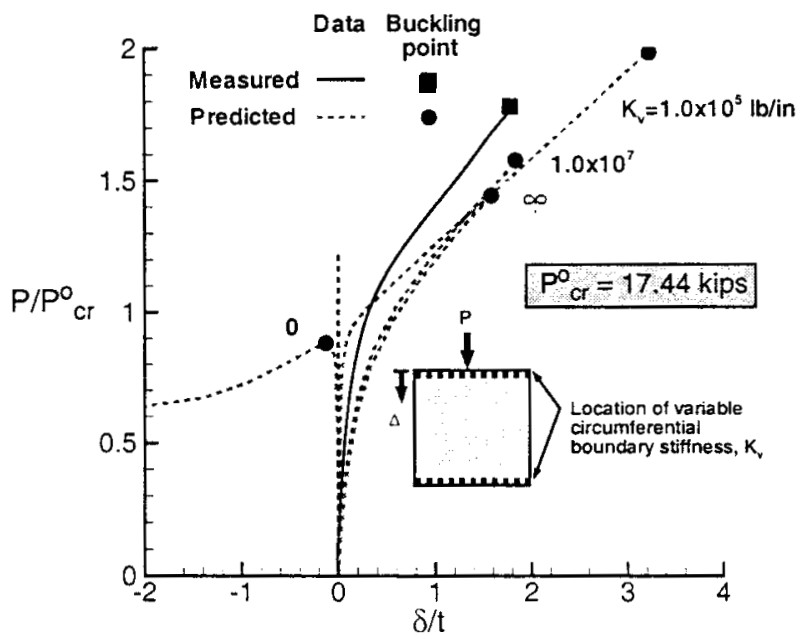

Fig. 24 Effects of circumferential boundary stiffness $K_{v}$ on the out-of-plane center displacement of a 60 -in-radius panel with $\mathrm{d} / \mathbf{W}=\mathbf{0}$.

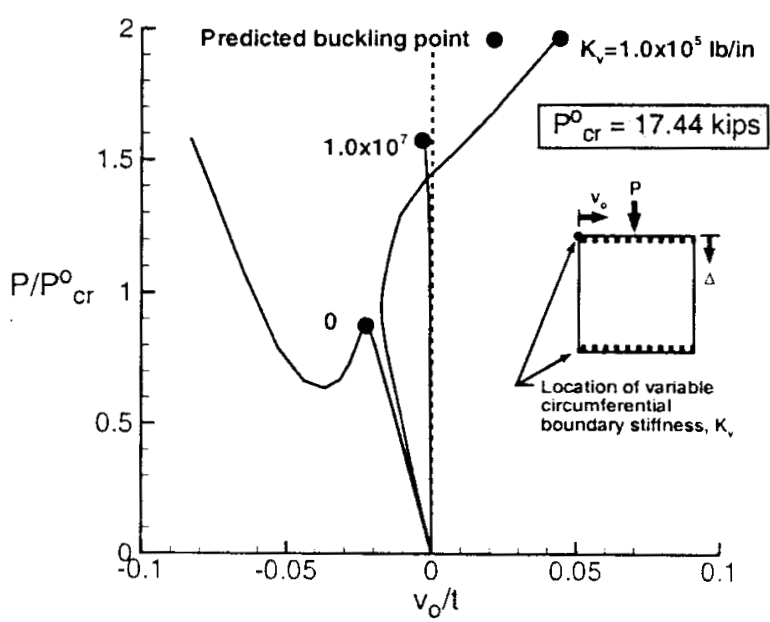

Fig. 25 liffects of circumferential boundary stiffness $K_{\text {v }}$ on the circumferential displacement of a corner of a 60 -in--inradius pancl with $d / W=0$. 


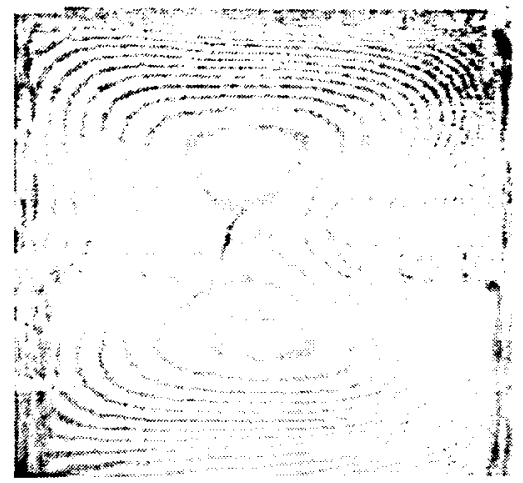

(a) Observed moirè fringe pattern,

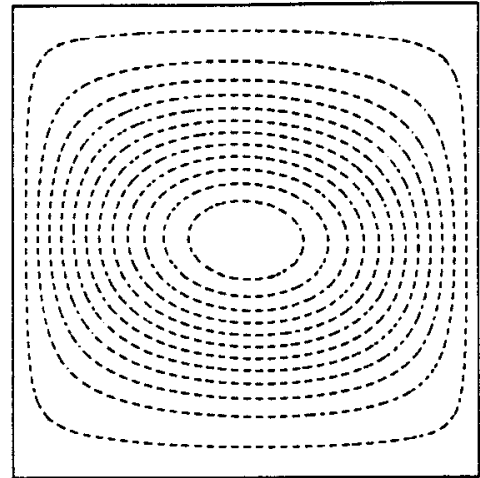

(b) $\mathrm{K}_{\mathrm{v}}=0, \mathrm{P} / \mathrm{P}_{\mathrm{cr}}=.87$

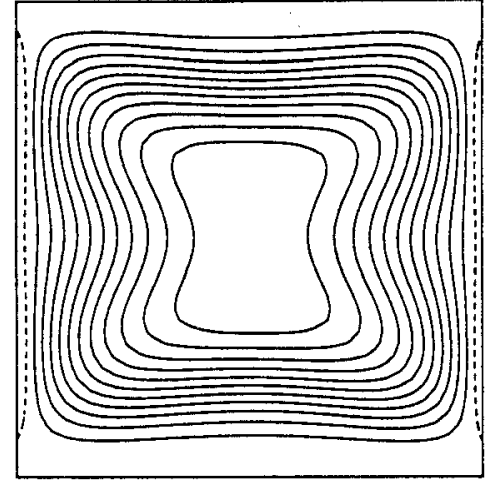

(c) $\mathrm{K}_{\mathrm{v}}=1.0 \times 10^{5}, \mathrm{P} / \mathrm{P}_{\mathrm{cr}}=1.93$ $\mathrm{P} / \mathbf{P}_{\mathrm{cr}}=\mathbf{1 . 7 5}$

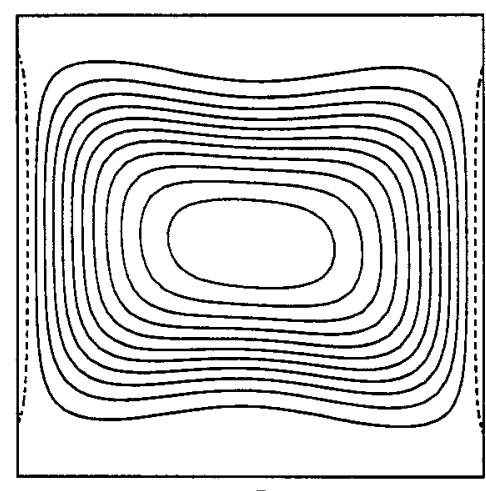

(d) $\mathrm{K}_{\mathrm{v}}=1.0 \times 10^{7}, \mathrm{P} / \mathrm{P}_{\mathrm{cr}}=1.56$

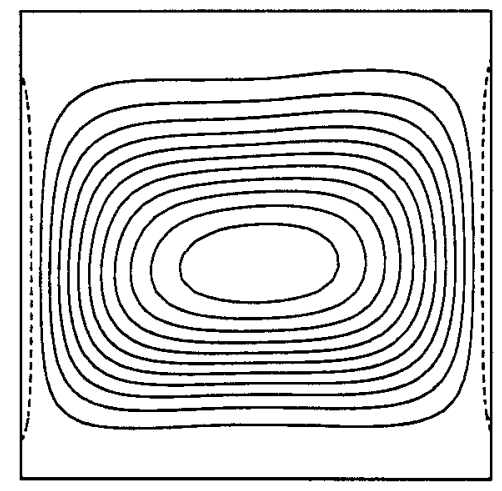

(e) $\mathrm{K}_{\mathrm{v}}=\infty, \mathrm{P} / \mathrm{P}_{\mathrm{cr}}=1.45$

Fig. 26 Predicted effects of circumferential boundary stiffness $K_{v}$ on the out-of-plane displacements at the onset of buckling of a 60 -in-radius panel with $d / W=0$ compared to observed out-of-plane displacements.

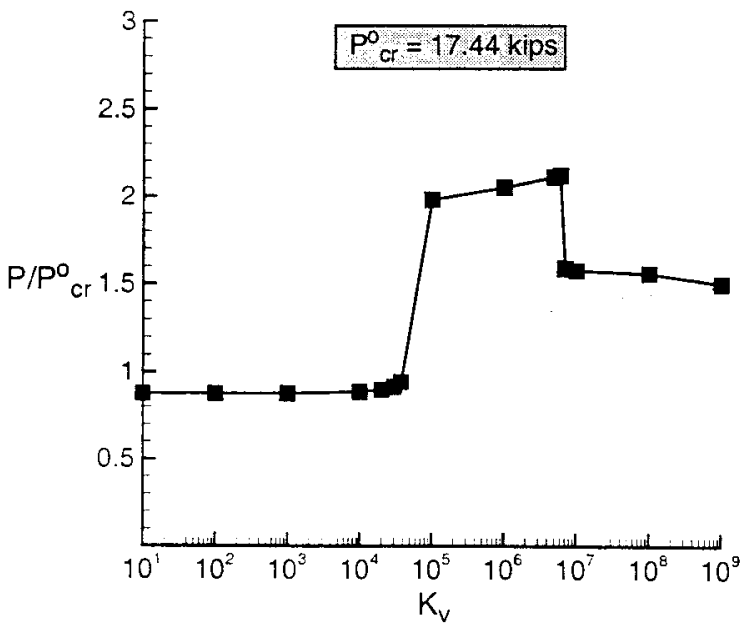

Fig. 27 Effects of circumferential boundary stiffness $K_{v}$ on the buckling load of a 60 -in-radius panel with $d / W=0$. 